\title{
Collapse and Revival of an Artificial Atom Coupled to a Structured Photonic Reservoir
}

\author{
Vinicius S. Ferreira $\odot,{ }^{1,2, *}$ Jash Banker $\odot,{ }^{1,2, *}$ Alp Sipahigil, ${ }^{1,2}$ Matthew H. Matheny, ${ }^{1,2}$ Andrew J. Keller, ${ }^{1,2}$ \\ Eunjong Kim $\odot,{ }^{1,2}$ Mohammad Mirhosseini, ${ }^{1,2}$ and Oskar Painter $\oplus^{1,2, \dagger}$ \\ ${ }^{1}$ Kavli Nanoscience Institute and Thomas J. Watson, Sr., Laboratory of Applied Physics, California \\ Institute of Technology, Pasadena, California 91125, USA \\ ${ }^{2}$ Institute for Quantum Information and Matter, California Institute of Technology, \\ Pasadena, California 91125, USA
}

(Received 9 March 2020; revised 12 September 2021; accepted 17 September 2021; published 2 December 2021)

\begin{abstract}
Quantum emitters in the presence of an electromagnetic reservoir with varying density of states, or structure, can undergo a rich set of dynamical behavior. In particular, the reservoir can be tailored to have a memory of past interactions with emitters, in contrast to memoryless Markovian dynamics of typical open systems. In this article, we investigate the non-Markovian dynamics of a superconducting qubit strongly coupled to a superconducting waveguide engineered to have both a sharp spectral variation in its transmission properties and a slowing of light by a factor of 650. Tuning the qubit into the spectral vicinity of the passband of this slow-light waveguide reservoir, we observe a 400-fold change in the emission rate of the qubit, along with oscillatory energy relaxation of the qubit resulting from the beating of bound and radiative dressed qubit-photon states. Furthermore, upon addition of a reflective boundary to one end of the waveguide, we observe revivals in the qubit population on a timescale 30 times longer than the inverse of the qubit's emission rate, corresponding to the round-trip travel time of an emitted photon. By in situ tuning of the qubit-waveguide interaction strength, we also probe a crossover between Markovian and nonMarkovian qubit emission dynamics in the presence of feedback from waveguide reflections. With this superconducting circuit platform, future studies of multiqubit interactions via highly structured reservoirs and the generation of multiphoton highly entangled states are possible.
\end{abstract}

DOI: 10.1103/PhysRevX.11.041043

Subject Areas: Metamaterials, Quantum Physics

\section{INTRODUCTION}

Spontaneous emission by a quantum emitter into the fluctuating electromagnetic vacuum, and the corresponding exponential decay of the emitter excited state, is an emblematic example of Markovian dynamics of an open quantum system [1]. However, modification of the electromagnetic reservoir can drastically alter this dynamic, introducing "non-Markovian" memory effects to the emission process, a consequence of information backflow from the reservoir to the emitter [2-5]. A canonical example of this, considered in early theoretical work [6-8], is the behavior of a quantum emitter whose natural emission frequency lies close to the gap edge of a photonic band-gap material $[9,10]$ where a sharp transition of the photonic density of states (DOS) occurs. Inside the band gap, the

\footnotetext{
*These authors contributed equally to this work. †opainter@caltech.edu; http://copilot.caltech.edu
}

Published by the American Physical Society under the terms of the Creative Commons Attribution 4.0 International license. Further distribution of this work must maintain attribution to the author(s) and the published article's title, journal citation, and DOI. emitter sees a reservoir devoid of electromagnetic states, while just outside of the band gap lies a continuum of states. This structure of the photonic band-gap reservoir leads to a strong dressing of the emitter and a resulting emission dynamics modified by the interplay between bound and radiative emitter-photon resonant states [11-15].

More recently, theoretical studies have explored how a structured reservoir with non-Markovian memory alters the entanglement within a quantum system coupled to such a reservoir [16-18]. This has led to the paradigm of reservoir engineering, where non-Markovianity is a quantifiable resource for quantum information processing and communication. Theory work from this quantum information perspective shows that long-lived reservoir correlations can be used for the generation and preservation of entanglement [19,20] and quantum control [21] of a quantum system, enhancement of the capacity of quantum channels [22], and the synthesis of exotic many-body quantum states of light from single emitters [23].

In practice, observation of non-Markovian emission phenomena can be achieved by strongly coupling an emitter to a single-mode waveguide- a one-dimensional (1D) reservoir with a continuum of states. Waveguides which break continuous translational symmetry, or which 
host resonant elements within the waveguide, are of particular interest in this regard owing to the structure in their spectrum [24-26]. For example, an array of coupled resonant elements leads to a constriction of the 1D continuum of guided modes to a transmission band of finite bandwidth, with sharp transitions in the photonic DOS occurring at the band edges as in a photonic band-gap material.

Spectral constriction of the waveguide continuum, and the concomitant frequency dispersion, can also result in the slowing of light propagation which enables observation of additional non-Markovian phenomena. For instance, by placing a reflective boundary (mirror) on one end of a slowlight waveguide, a fraction of the emitter's radiation can be fed back from the waveguide reservoir to the emitter at significantly delayed timescales [27-29]. The nonMarkovian regime is reached when $\tau_{d} \Gamma_{1 \mathrm{D}}>1$, where $\Gamma_{1 \mathrm{D}}$ is the emitter's emission rate into the waveguide and $\tau_{d}$ is the round-trip travel time of an emitted photon. Theoretical studies show that such non-Markovian timedelayed feedback in a 1D waveguide reservoir can lead to revivals in the excited-state population of an emitter as it undergoes spontaneous emission decay [27,30-35], realization of stable bound states in a continuum [36,37], and enhanced collective effects including multipartite entanglement and superradiant emission from emitters interacting via a common waveguide channel [18,38-42]. This deceptively simple mechanism of time-delayed feedback can also be used for the generation of multidimensional photonic cluster states by a single emitter and has been proposed as a means for generating the universal resource states necessary for measurement-based quantum computation [23].

Superconducting microwave circuits incorporating Josephson-junction-based qubits $[43,44]$ represent a nearideal test bed for studying the quantum dynamics of emitters interacting with a $1 \mathrm{D}$ continuum $[45,46]$. In comparison to solid-state and atomic optical systems [47-50], superconducting microwave circuits can be created at a deep-subwavelength scale, giving rise to strong qubit-waveguide coupling far exceeding other qubit dissipative channels. This has enabled a variety of pioneering experiments probing qubit-waveguide radiative dynamics, employing waveguide spectroscopy [29,51-53], timedependent qubit measurements [54-57], and analysis of higher-order field correlations [58,59]. Recent experiments also explore the coupling of superconducting qubits to acoustic wave devices, demonstrating the capability of these systems to produce significant time-delayed feedback and remote entanglement of qubits $[53,57]$.

In this work, we present the design and characterization of an all-electrical slow-light waveguide consisting of a chain of coupled lumped-element superconducting resonators patterned on a silicon microchip. We demonstrate that this compact, low-loss microwave waveguide has sharp band edges and a passband with group delay of 55 ns per centimeter over an $80-\mathrm{MHz}$ bandwidth. Through the addition of strongly coupled Xmon-style superconducting qubits $[60,61]$ to the slow-light waveguide, we are able to realize a quantum emitter-reservoir system operating deep within the non-Markovian limit. Spectroscopic measurement of the coupled system shows the emergence of dressed qubit-photon resonant states near the band edges of the constricted passband of the waveguide [7,8,52]. Using nonadiabatic tuning of the qubit emission frequency, we also measure the time-dependent dynamics of the qubit excited-state population when it is resonant at different points across the band gap and passband of the waveguide. We directly observe nonexponential, oscillatory radiative decay of the qubit, which modeling indicates is a result of the interference of the pair of bound and radiative dressed qubit-photon states that exist on either side of the band edge of the slow-light waveguide [11]. Furthermore, by terminating one end of the slow-light waveguide with a reflective boundary, we explore the effects of time-delayed feedback on the qubit emission as it emits into the passband of the slow-light waveguide. In this regime, we observe multiple, well-resolved revivals in the qubit excited-state population and explore the crossover between Markovian and nonMarkovian emission dynamics through in situ tuning of the qubit coupling to the waveguide. From this series of measurements, we estimate the achievable fidelity of entangling a number of photon pulses via qubit emission and subsequent time-delayed feedback and find that the demonstrated qubit-waveguide system is a promising platform for the sequential generation of multidimensional photonic cluster states as described in the theoretical proposals of Refs. [23,62-64].

\section{SLOW-LIGHT METAMATERIAL WAVEGUIDE}

In prior work studying superconducting qubit emission into a photonic band-gap waveguide [54], we employed a metamaterial consisting of a coplanar waveguide (CPW) periodically loaded by lumped-element resonators. In that geometry, whose circuit model simplifies to a transmission line with resonator loading in parallel to the line, one obtains high-efficiency transmission with a characteristic impedance approximately that of the standard CPW away from the resonance frequency of the loading resonators and a transmission stop band near resonance of the resonators. The spectral characteristics of the metamaterial in Ref. [54] were studied via spontaneous emission lifetime and Lambshift measurements of a weakly coupled superconducting qubit, which revealed information about the local DOS at the qubit frequency that was consistent with the metamaterial engineered dispersion. In contrast, here we seek a waveguide with high transmission efficiency, slow-light propagation within a transmission passband, and considerably stronger qubit coupling to the waveguide-guided modes. The stronger coupling renders the Born approximation inapplicable in such a system, where the effect of 
the qubit interaction with the photonic reservoir takes on significantly more complexity than simply a decay rate dependent solely on the DOS at the qubit frequency. Furthermore, the increased propagation delay gives rise to non-Markovian memory effects in the waveguide-mediated interactions between qubits, for which the waveguide degrees of freedom can no longer be traced out, as in Ref. [55], for instance.

Large delay per unit area can be obtained by employing a network of subwavelength resonators, with light propagation corresponding to hopping from resonator to resonator at a rate set by near-field interresonator coupling. This areaefficient approach to achieving large delays is well suited to applications where only limited bandwidths are necessary. However, realizing such a waveguide system in a compact chip-scale form factor requires a modular implementation that can be reliably replicated at the unit-cell level without introducing spurious cell-to-cell couplings. In optical photonics applications, this sort of scheme is realized in what are called coupled-resonator optical waveguides, or CROW waveguides $[65,66]$. Here, we employ a periodic array of capacitively coupled, lumped-element microwave resonators to form the waveguide. Such a resonator-based waveguide supports a photonic channel through which light can propagate, henceforth referred to as the passband, with bandwidth approximately equal to 4 times the coupling between the resonators, $J$. The limited bandwidth directly translates into large propagation delays; as can be shown (see the Appendix B), the delay in the resonator array is roughly $\omega_{0} / J$ longer than that of a conventional CPW of similar area, where $\omega_{0}$ is the resonance frequency of the resonators.

Optical and scanning electron microscope (SEM) images of the unit cell of the metamaterial slow-light waveguide used in this work are shown in Fig. 1(a). The cell consists of a tightly meandered wire inductor section $\left(L_{0}\right.$; false color blue) and a top shunting capacitor $\left(C_{0}\right.$; false color green), forming the lumped-element microwave resonator. Note that these delineations between inductor and capacitor are not strict and that the meandered wire inductor (top shunting capacitor) has a small parasitic capacitance (parasitic inductance). The resonator is surrounded by a large ground plane (gray) which shields the meander wire section. Laterally extended "wings" of the top shunting capacitor also provide coupling between the cells $\left(C_{g}\right.$; false color green). Note that at the top of the optical image, above each shunting capacitor, we include a long superconducting island $\left(C_{q}\right.$; false color green); this is used in the next section as the shunting capacitance for Xmon qubits. Similar lumped-element resonators have been realized with internal quality factors of $Q_{i} \sim 10^{5}$ and small resonator frequency disorder [54], enabling propagation of light with low extinction from losses or disorder-induced scattering [67]. The waveguide resonators shown in Fig. 1(a) have a bare resonance frequency of $\omega_{0} / 2 \pi \approx 4.8 \mathrm{GHz}$, unit-cell length (a)

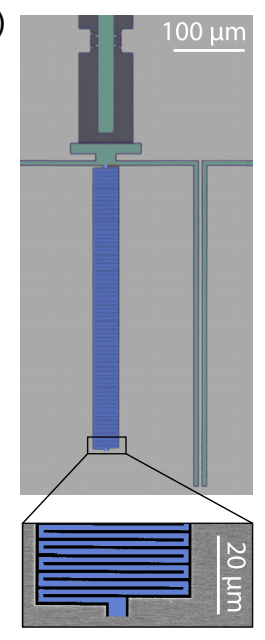

(b)

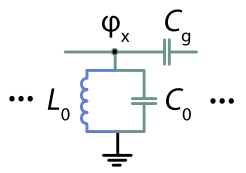

(c)

(d)
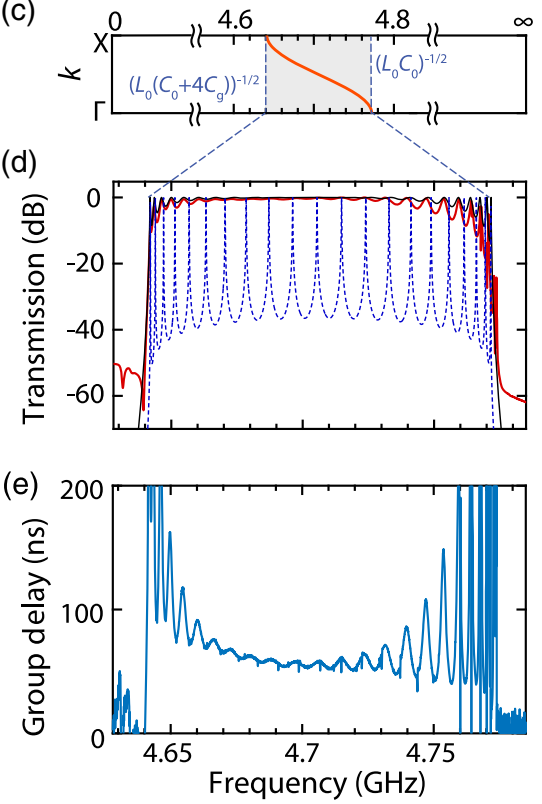

FIG. 1. Microwave coupled resonator array slow-light waveguide. (a) Optical image of a fabricated microwave resonator unit cell. The capacitive elements of the resonator are false colored in green, while the inductive meander is false colored in blue. The inset shows a false-colored SEM image of the bottom of the meander inductor, where it is shorted to ground. (b) Circuit diagram of the unit cell of the periodic resonator array waveguide. (c) Theoretical dispersion relation of the periodic resonator array. See Appendix B for the derivation. (d) Transmission through a metamaterial slow-light waveguide spanning 26 resonators and connected to $50-\Omega$ input-output ports. Dashed blue line: theoretical transmission of finite array without matching to $50-\Omega$ boundaries. Black line: theoretical transmission of finite array matched to $50-\Omega$ boundaries through two modified resonators at each boundary. Red line: measured transmission for a fabricated finite resonator array with boundary matching to input-output $50-\Omega$ coplanar waveguides. The measured ripple in transmission is less than $0.5 \mathrm{~dB}$ in the middle of the passband. (e) Measured group delay $\tau_{g}$. Ripples in $\tau_{g}$ are less than $\delta \tau_{g}=5 \mathrm{~ns}$ in the middle of the passband.

$d=290 \mu \mathrm{m}$, and transverse unit-cell width $w=540 \mu \mathrm{m}$, achieving a compact planar form factor of $\bar{d} / \lambda=(\sqrt{d w}) /$ $\left(2 \pi v / \omega_{0}\right) \approx 1 / 60$, where $v$ is the speed of light in a CPW on an infinitely thick silicon substrate.

The unit cell is to a good approximation given by the electrical circuit shown in Fig. 1(b), in which the photon hopping rate is $J \propto C_{g} / C_{0}$ [68]. We choose a ratio of $C_{g} / C_{0} \approx 1 / 70$, which yields a delay per resonator of roughly 2 ns. Note that we achieve this compact form factor and large delay per resonator while separating different lumped-element components by large amounts of ground plane, which minimizes spurious cross talk between different unit cells. Analysis of the periodic circuit's Hamiltonian and dispersion can be found in Appendix B, where the dispersion is shown to be 
$\omega_{k}=\omega_{0} / \sqrt{1+4\left(C_{g} / C_{0}\right) \sin ^{2}(k d / 2)}$. Figure 1(c) shows a plot of the theoretical waveguide dispersion for an infinitely periodic waveguide, where the frequency of the band edges of the passband are denoted with the circuit parameters of the unit cell.

For finite resonator arrays, care must be taken to avoid reflections at the boundaries that would result in spurious resonances [see Fig. 1(d), dashed blue curve, for example]. To avoid these reflections, we taper the impedance of the waveguide by slowly shifting the capacitance of the resonators at the boundaries. In particular, we modify the first two unit cells at each boundary, but, in principle, more resonators could have been modified for a more gradual taper. Increasing $C_{g}$ to increase the coupling between resonators, and decreasing $C_{0}$ to compensate for resonance frequency changes, effectively impedance matches the Bloch impedance of the periodic structure in the passband to the characteristic impedance of the inputoutput waveguides [69]. In essence, this tapering achieves strong coupling of all normal modes of the finite structure to the input-output waveguides by adiabatically transforming guided resonator array modes into guided input-output waveguide modes. This loading of the normal modes lowers their $Q$ such that they spectrally overlap and become indistinguishable, changing the DOS of a finite array from that of a multimode resonator to that of finite-bandwidth continuum with singular band edges. Further details of the design of the unit cell and boundary resonators can be found in Appendix C.

Using the above design principles, we fabricated a capacitively coupled 26-resonator array metamaterial waveguide. The waveguide is fabricated using electronbeam deposited aluminum $(\mathrm{Al})$ on a silicon substrate and is measured in a dilution refrigerator; transmission measurements are shown in Figs. 1(d) and 1(e), and further details of our fabrication methods and measurement setup can be found in Appendix A. We find less than $0.5 \mathrm{~dB}$ ripple in transmitted power and less than $10 \%$ variation in the group delay $\left(\tau_{g} \equiv-(d \phi / d \omega), \phi=\arg [t(\omega)]\right.$, where $t$ is transmission) across $80 \mathrm{MHz}$ of bandwidth in the center of the passband, ensuring low distortion of propagating signals. Qualitatively, this small ripple demonstrates that we have realized a resonator array with small disorder and precise modification of the boundary resonators. More quantitatively, from the transmitted power measurements, we extract a standard deviation in the resonance frequencies of $3 \times 10^{-4} \times \omega_{0}$ (see Appendix D). Furthermore, we achieve $\tau_{d} \approx 55 \mathrm{~ns}$ of delay across the 1 -cm metamaterial waveguide, corresponding to a slow-down factor given by the group index of $n_{g} \approx 650$. We stress that this group delay is obtained across the center of the passband rather than near the band edges where large (and undesirable) higher-order dispersion occurs concomitantly with large delays.

\section{NON-MARKOVIAN RADIATIVE DYNAMICS}

In order to study the non-Markovian radiative dynamics of a quantum emitter, a second sample is fabricated with a metamaterial waveguide similar to that in the previous section, this time including three flux-tunable Xmon qubits [61] coupled at different points along the waveguide [see Figs. 2(a)-2(c)]. Each of the qubits is coupled to its own $X Y$ control line for excitation of the qubit, a $Z$ control line for flux tuning of the qubit transition frequency, and a readout resonator $(R)$ with separate readout waveguide (RO) for dispersive readout of the qubit state. The qubits are designed to be in the transmon limit [60] with large tunneling to charging energy ratio (see Refs. [54,70] for further qubit design and fabrication details). As in the test waveguide in Fig. 1, the qubit-loaded metamaterial waveguide is impedance matched to input-output $50-\Omega$ CPWs. In order to extend the waveguide delay further, however, this new waveguide is realized by concatenating two of the test metamaterial waveguides together using a CPW bend and internal impedance-matching sections. The Xmon qubit capacitors are designed to have capacitive coupling to a single unit cell of the metamaterial waveguide, yielding a qubit-unit-cell coupling of $g_{\mathrm{uc}} \approx 0.8 \mathrm{~J}$.

In this work, only one of the qubits, $Q_{1}$, is used to probe the non-Markovian emission dynamics of the qubit-waveguide system. The other two qubits are to be used in a separate experiment and are detuned from $Q_{1}$ by approximately $1 \mathrm{GHz}$ for all of the measurements that follow. At zero flux bias (i.e., maximum qubit frequency), the measured parameters of $Q_{1}$ are $\omega_{g e} / 2 \pi=$ $5.411 \mathrm{GHz}, \eta / 2 \pi=\left(\omega_{e f}-\omega_{g e}\right) / 2 \pi=-235 \mathrm{MHz}, \omega_{r} / 2 \pi=$ $5.871 \mathrm{GHz}$, and $g_{r} / 2 \pi=88 \mathrm{MHz}$. Here, $|g\rangle,|e\rangle$, and $|f\rangle$ are the vacuum, first excited, and second excited states of the Xmon qubit, respectively, with $\omega_{g e}$ the fundamental qubit transition frequency, $\omega_{e f}$ the first excitedstate transition frequency, and $\eta$ the anharmonicity. $\omega_{r}$ is the readout resonator frequency, and $g_{r}$ is the bare coupling rate between the qubit and the readout resonator.

As an initial probe of qubit radiative dynamics, we spectroscopically probe the interaction of $Q_{1}$ with the structured 1D continuum of the metamaterial waveguide. These measurements are performed by tuning $\omega_{g e}$ into the vicinity of the passband and measuring the waveguide transmission spectrum at low power (such that the effects of qubit saturation can be neglected). A color-intensity plot of the measured transmission spectrum versus flux bias used to tune the qubit frequency is displayed in Fig. 2(d). These spectra show a clear anticrossing as the qubit is tuned toward either band edge of the passband [an enlargement near the upper band edge of the passband is shown in Fig. 2(e)]. As shown theoretically [11,12], in the singleexcitation manifold, the interaction of the qubit with the waveguide results in a pair of qubit-photon dressed states of the hybridized system, with one state in the passband (a delocalized "continuum" state) and one state in the band 
(a)

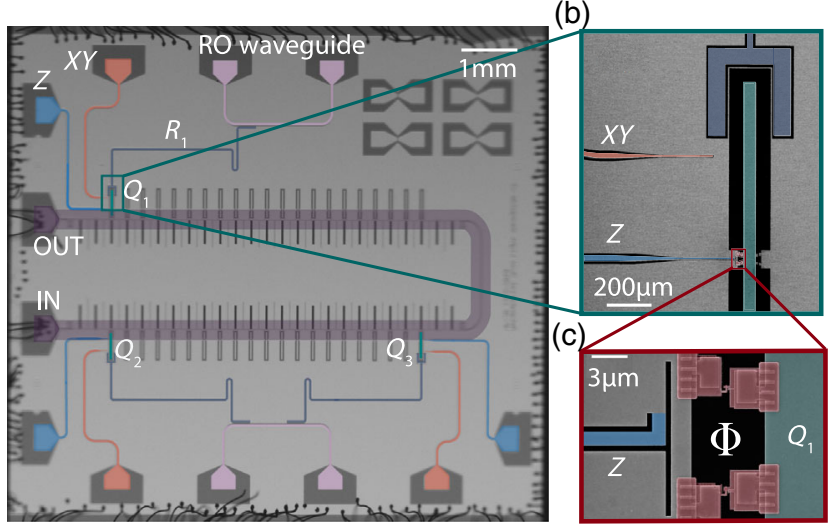

(d)

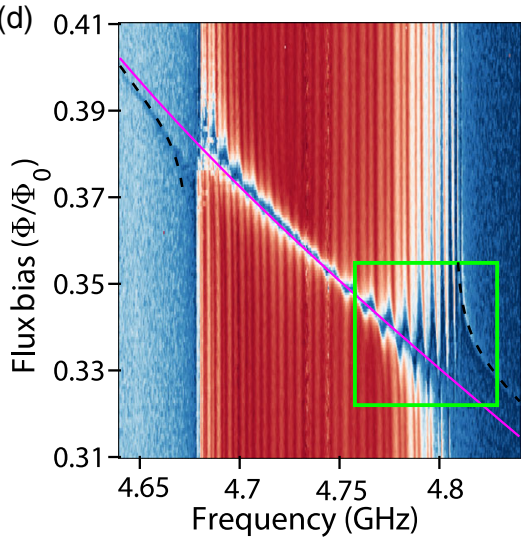

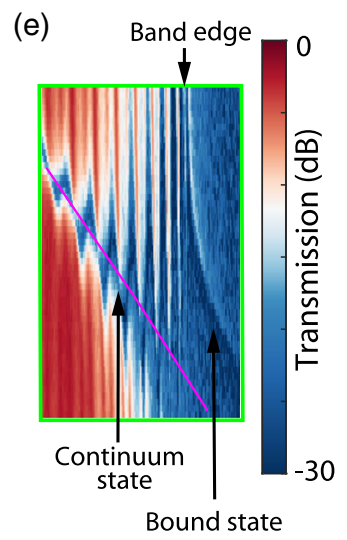

FIG. 2. Artificial atom coupled to a structured photonic reservoir. (a) False-colored optical image of a fabricated sample consisting of three transmon qubits $\left(Q_{1}, Q_{2}\right.$, and $\left.Q_{3}\right)$ coupled to a slow-light metamaterial waveguide composed of a coupled microwave resonator array. Each qubit is capacitively coupled to a readout resonator (false-color dark blue) and an $X Y$ control line (false-color red) and inductively coupled to a $Z$ flux line for frequency tuning (false-color light blue). The readout resonators are probed through feedlines (false-color lilac). The metamaterial waveguide path is highlighted in false-color dark purple. (b) SEM image of the $Q_{1}$ qubit, showing the long, thin shunt capacitor (false-color green), $X Y$ control line, the $Z$ flux line, and coupling capacitor to the readout resonator (falsecolor dark blue). (c) SEM enlarged image of the $Z$ flux line and superconducting quantum interference device (SQUID) loop of the $Q_{1}$ qubit, with Josephson junctions and its pads false colored in crimson. (d) Transmission through the metamaterial waveguide as a function of the flux. The solid magenta line indicates the expected bare qubit frequency in the absence of coupling to the metamaterial waveguide, calculated based on the measured qubit minimum and maximum frequencies and the extracted anharmonicity. The dashed black lines are numerically calculated bound-state energies from a model Hamiltonian of the system; see Appendix E for further details. (e) Enlargement of transmission near the upper band edge, showing the hybridization of the qubit with the band edge, and its decomposition into a bound state in the upper band gap and a radiative state in the continuum of the passband.

gap (a localized "bound" state). This arises due to the large peak in the photonic DOS at the band edge (in the lossless case, a van Hove singularity), the modes of which strongly couple to the qubit with a coherent interaction rate of $\Omega_{\mathrm{WG}} \approx\left(g_{\mathrm{uc}}^{4} / 4 J\right)^{1 / 3}$, resulting in a dressed-state splitting of $2 \Omega_{\mathrm{WG}}$. This splitting has been experimentally shown to be a spectroscopic signature of a non-Markovian interaction between an emitter and a photonic crystal reservoir [51,52]. Further details and discussion can be found in Appendixes B and E.

The dressed state with frequency in the passband is a radiative state which is responsible for decay of the qubit into the continuum [8]. On the other hand, the state with frequency in the gap is a qubit-photon bound state, where the qubit is self-dressed by virtual photons that are emitted and reabsorbed due to the lack of propagating modes in the waveguide for the radiation to escape. This bound state assumes an exponentially shaped photonic wave function of the form $\sum_{x} e^{-|x| / \lambda} \hat{a}_{x}^{\dagger} \mid$ vac $\rangle$, where $\mid$ vac $\rangle$ is the state with no photons in the waveguide, $\hat{a}_{x}^{\dagger}$ is the creation operator of a photon in unit cell at position $x$ (with the qubit located at $x=0$ ), and $\lambda \approx \sqrt{J /\left(E_{b}-\omega_{0}\right)}$ is the state's localization length. In the theoretical limit of an infinite array, and in the absence of intrinsic resonator and qubit losses, the qubit component of the bound state does not decay even though it is hybridized with the waveguide continuum, a behavior distinct from conventional open quantum systems. Practically, however, intrinsic losses and the overlap between the bound state's photonic wave function and the input-output waveguides results in decay of the qubit-photon bound state.

In complement to spectroscopic probing of the qubitreservoir system and in order to directly study the population dynamics of the qubit-photon dressed states, we also perform time-domain measurements as shown in Fig. 3. In this protocol [illustrated in Fig. 3(a)], we excite the qubit to state $|e\rangle$ with a resonant $\pi$ pulse on the $X Y$ control line and then rapidly tune the qubit transition frequency using a fast current pulse on the $Z$ control line to a frequency $\left(\omega_{g e}^{\prime}\right)$ within, or in the vicinity of, the slowlight waveguide passband. After an interaction time $\tau$, the qubit is then rapidly tuned away from the passband, and the remaining qubit population in $|e\rangle$ is measured using a microwave probe pulse (RO) of the readout resonator which is dispersively coupled to the qubit. The excitation of the qubit is performed far from the passband, permitting initialization of the transmon qubit while it is negligibly hybridized with the guided modes of the waveguide. Dispersive readout of the qubit population is performed outside of the passband in order to minimize the loss of population during readout. Note that, as illustrated in Fig. 3(a), the qubit is excited and measured at different frequencies on opposite sides of the passband; this is necessary to avoid Landau-Zener interference [71].

Results of measurements of the time-domain dynamics of the qubit population as a function of $\omega_{g e}^{\prime}$ (the estimated 
(a)

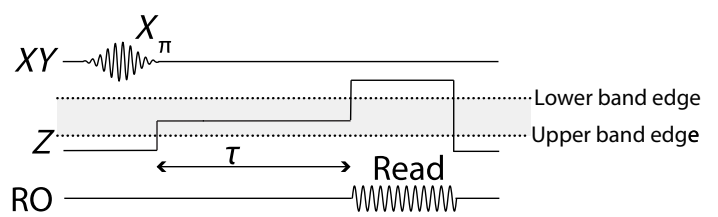

(b)

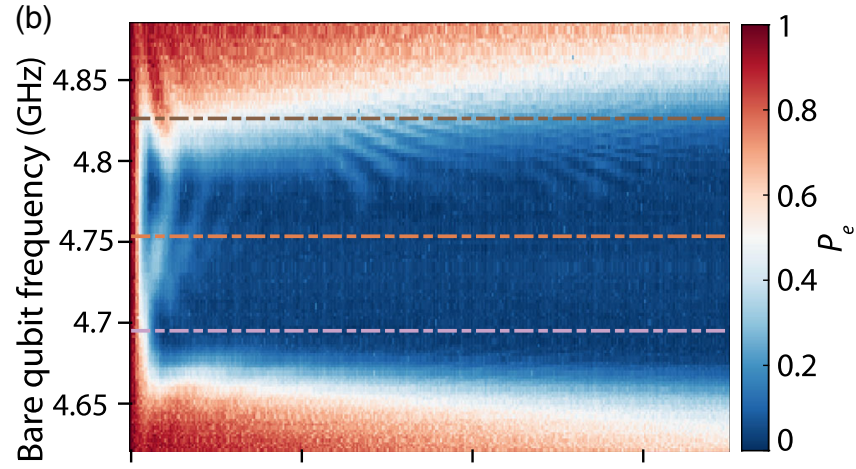

(c)

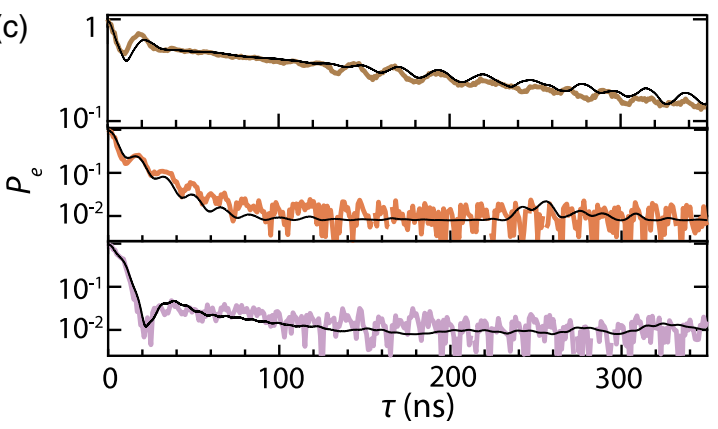

FIG. 3. Non-Markovian radiative dynamics in a structured photonic reservoir. (a) Pulse sequence for the time-resolved measurement protocol. The qubit is excited while its frequency is $250 \mathrm{MHz}$ above the upper band edge, and then it is quickly tuned to the desired frequency $\left(\omega_{g e}^{\prime}\right)$ for a interaction time $\tau$ with the reservoir. After interaction, the qubit is quickly tuned below the lower band edge for dispersive readout. (b) Intensity plot showing the excited-state population of the qubit versus interaction time with the metamaterial waveguide reservoir as a function of the bare qubit frequency. (c) Line cuts of the intensity plot shown in (b), where the color of the plotted curve matches the corresponding horizontal dot-dashed curve in the intensity plot. Solid black lines are numerical predictions of a model with experimentally fitted device parameters and an assumed $0.8 \%$ thermal qubit population (see Appendix E for further details).

bare qubit frequency during interaction with the waveguide) are shown as a color-intensity plot in Fig. 3(b). In this plot, we observe a 400-fold decrease in the 1 / $e$ excitedstate lifetime of the qubit as it is tuned from well outside the passband to the middle of the slow-light waveguide passband, reaching a lifetime as short as $7.5 \mathrm{~ns}$. Beyond the large change in qubit lifetime within the passband, several other more subtle features can be seen in the qubit population dynamics near the band edges and within the passband. These more subtle features in the measured dynamics show nonexponential decay, with significant oscillations in the excited-state population that is a hallmark of strong non-Markovianity in quantum systems coupled to amplitude damping channels [72,73].

The observed qubit emission dynamics in this nonMarkovian limit are best understood in terms of the qubit-waveguide dressed states. Fast (i.e., nonadiabatic) tuning of the qubit in state $|e\rangle$ into the proximity of the passband effectively initializes it into a superposition of the bound and continuum dressed states. The observed earlytime interaction dynamics of the qubit with the waveguide then originate from interference of the dressed states, which leads to oscillatory behavior in the qubit population analogous to vacuum-Rabi oscillations [74]. The frequency of these oscillations is thus set by the difference in energy between the dressed states. The amplitude of the oscillations, on the other hand, quickly decay away as the energy in the radiative continuum dressed state is lost into the waveguide.

All of these features can be seen in Fig. 3(c), which shows plots of the measured time-domain curves of the qubit excited-state population for bare qubit frequencies near the top, middle, and bottom of the passband. Near the upper band-edge frequency, we observe an initial oscillation period as expected due to dressed-state interference. Once the continuum dressed state decays away, a slower decay region free of oscillations can be observed (this is due to the much slower decay of the remaining qubitphoton bound state). Finally, around $\tau \approx 115 \mathrm{~ns}$, there is an onset of further small-amplitude oscillations in the qubit population. These late-time oscillations can be attributed to interference of the remaining bound state at the site of the qubit with weak reflections occurring within the slow-light waveguide of the initially emitted continuum dressed state. The 115-ns timescale corresponds to the round-trip time between the qubit and the CPW bend that connects the two slow-light waveguide sections.

In the middle of the passband, we see an extended region of initial oscillation and rapid decay, albeit of smaller oscillation amplitude. This is a result of the much smaller initial qubit-photon bound-state population when tuned to the middle of the passband. Near the bottom of the passband, we see rapid decay and a single period of a much slower oscillation. This is curious, as the dispersion near the upper and lower band-edge frequencies of the slow-light waveguide is nominally equivalent. Further modeling shows that this is a result of weak nonlocal coupling of the Xmon qubit to a few of the nearest-neighbor unit cells of the waveguide. Referring to Fig. 1(c), the modes near the lower band edge occur at the $X$ point of the Brillouin zone edge, where the modes have alternating phases across each unit cell; thus, extended coupling of the Xmon qubit causes cancellation effects which reduce the qubit-waveguide coupling at the lower-frequency band edge. Further detailed numerical model simulations of our qubit-waveguide system via a tight-binding model and a circuit model, as well as the correspondence between the observed dynamics and 
the theory of spontaneous emission by a two-level system near a photonic band edge [11], are given in Appendix E.

\section{TIME-DELAYED FEEDBACK}

In order to further study the late-time, non-Markovian memory effects of the qubit-waveguide dynamics, we also perform measurements in which the end of the waveguide furthest from qubit $Q_{1}$ is terminated with an open circuit, effectively creating a "mirror" for photon pulses stored in the slow-light waveguide reservoir. As illustrated in Fig. 4(a), we achieve this in situ by connecting the input microwave cables of the dilution refrigerator to the waveguide via a microwave switch. The position of the switch, electrically closed or open, allows us to study a truly open environment for the qubit or one in which delayed feedback is present, respectively (see Appendix A for further details).

Performing time-domain measurements with the mirror in place and with the qubit frequency in the passband, we observe recurrences in the qubit population at 1 and 2 times the round-trip time of the slow-light waveguide that do not appear in the absence of the mirror [see Fig. 4(b)]. The separation of timescales between full population decay of the qubit and its time-delayed reexcitation demonstrates an exceptionally long memory of the reservoir due to its slow-light nature and places this experiment in the deep non-Markovian regime [27]. The small recurrence levels as they appear in Fig. 4(b) are not due to inefficient mirror reflection but rather can be explained as follows. Because the qubit emits toward both ends of the waveguide, half of the emission is lost to the unterminated end, while the other half is reflected by the mirror and returns to the qubit. In addition, the exponentially decaying temporal profile of the emission leads to inefficient reabsorption by the qubit and further limits the recurrence (see, for instance, Refs. [75,76] for details). These two effects can be observed in simulations of a qubit coupled to a dispersionless and lossless waveguide (pink dotted line; for more details, see Ref. [31] and Appendix G). The remaining differences between the simulation and the measured population recurrence (blue solid line) can be explained by the effects of propagation loss and pulse distortion due to the slow-light waveguide's dispersion.

We also further probe the dependence of this phenomenon on the strength of coupling to the waveguide continuum by parametric flux modulation of the qubit transition frequency [77] when it is far detuned from the passband. This modulation creates sidebands of the qubit excited state, which are detuned from $\omega_{g e}$ by the frequency of the flux tone $\omega_{\text {mod }}$. By choosing the modulation frequency such that a first-order sideband overlaps with the passband, the effective coupling rate of the qubit with the waveguide at the sideband frequency is reduced approximately by a factor of $\mathcal{J}_{1}^{2}\left[\epsilon / \omega_{\text {mod }}\right]$, where $\epsilon$ is

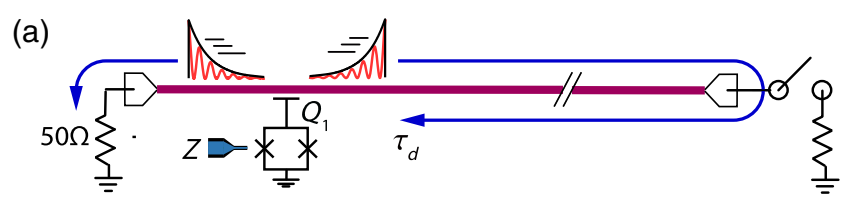

(b)
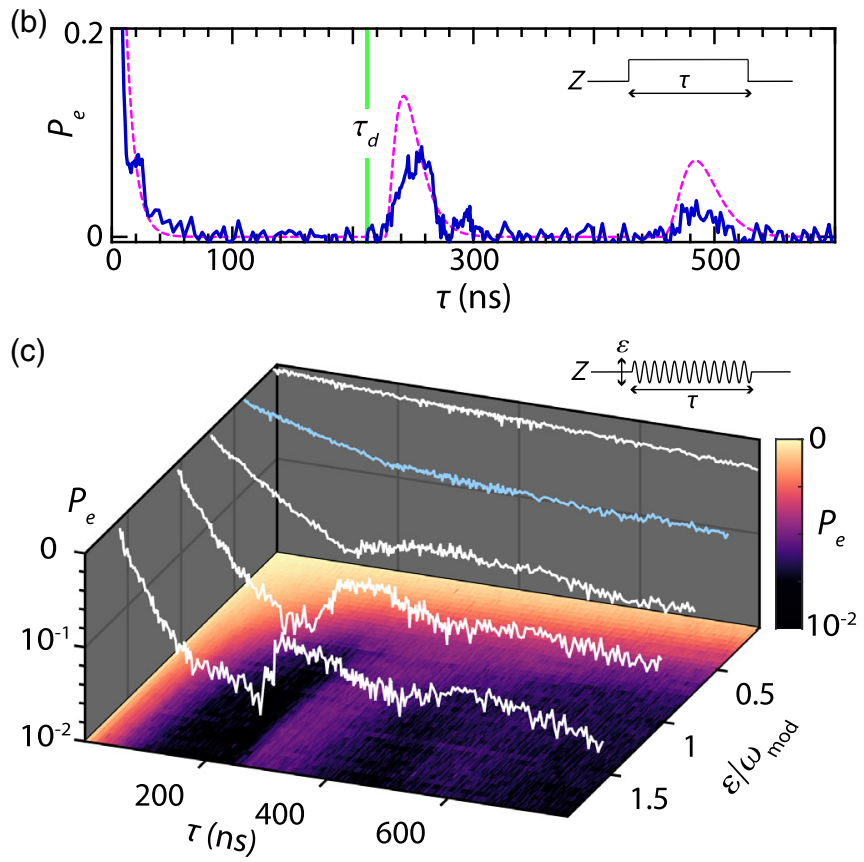

FIG. 4. Time-delayed feedback from a slow-light reservoir with a reflective boundary. (a) Illustration of the experiment, showing the qubit coupled to the metamaterial waveguide which is terminated on one end with a reflective boundary via a microwave switch. (b) Measured population dynamics of the excited state of the qubit when coupled to the metamaterial waveguide terminated in a reflective boundary. Here, the bare qubit is tuned into the middle of the passband. The onset of the population revival occurs at $\tau=227 \mathrm{~ns}$, consistent with round-trip group delay $\left(\tau_{d}\right)$ measurements at that frequency, while the emission lifetime of the qubit is $\left(\Gamma_{1 \mathrm{D}}\right)^{-1}=7.5 \mathrm{~ns}$. The magenta curve is a theoretical prediction for emission of a qubit into a dispersionless, lossless semi-infinite waveguide with equivalent $\tau_{d}$ and $\Gamma_{1 \mathrm{D}}$ (see Appendix $\mathrm{G}$ for details). (c) Population dynamics under parametric flux modulation of the qubit, for varying modulation amplitudes, demonstrating a Markovian to non-Markovian transition. When the modulation index $\left(\epsilon / \omega_{\text {mod }}\right)$ is approximately 0.4 , we have $\Gamma_{1 \mathrm{D}}(\epsilon)=1 / \tau_{d}$; the corresponding dynamical trace is colored in blue.

the modulation amplitude and $\mathcal{J}_{1}$ is a Bessel function of the first kind $\left(\epsilon / \omega_{\bmod }\right.$ is the modulation index). Keeping a fixed $\omega_{\text {mod }}$, we observe purely exponential decay at small modulation amplitudes. However, above a modulation amplitude threshold, we again observe recurrences in the qubit population at the round-trip time of the metamaterial waveguide, demonstrating a continuous transition from Markovian to non-Markovian dynamics (see Appendix G for further comparisons between these data and the theoretical model in Ref. [31]). 


\section{CONCLUSION}

In conclusion, by strongly coupling Xmon qubits to a 1D structured photonic reservoir consisting of a metamaterial slow-light waveguide, we are able to probe the nonMarkovian dynamical regime of waveguide quantum electrodynamics. In this regime, we observe nonexponential qubit spontaneous decay near the band edges of the slow-light waveguide, attributable to interference resulting from the splitting of the qubit state into a radiative state in the passband and a bound state in the band-gap region of the metamaterial waveguide. Moreover, by placing a reflective boundary on one end of the waveguide, we observe recurrences in the qubit population at the round-trip time of an emitted photon, as well as a Markovian to non-Markovian transition when varying the qubit-waveguide interaction strength.

The demonstrated ability to achieve a true finitebandwidth continuum with time-delayed feedback opens up several new research avenues for exploration [28,3042,78]. As a straightforward extension of the current work, one may probe the qubit-waveguide-mirror system in a continuous, strongly driven fashion and use tomography to study photon correlations in the output radiation field [28]. This output field, with an expected photon stream of high entanglement dimensionality, has a direct mapping to continuous matrix product states which can used for analog simulations of higher-dimension interacting quantum fields $[78,79]$. With technical advancements in the tomography of microwave fields $[59,80]$ and realization of single-microwave-photon qubit detectors [81-83], the basic tools for characterization of these entangled photonic states and their quantum many-body-system analogues are now available.

Looking forward even further, the use of the multilevel structure of the transmon qubit, in conjunction with a second distant qubit side coupled to the waveguide as a switchable mirror, can be used to generate 2D cluster states [23]. This system is capable of entangling consecutively emitted photons as well as photons separated in time by the round-trip waveguide delay $\tau_{d}$, thus achieving an $N \times M$ 2D cluster state, where $N$ is limited by the number of nonoverlapping photons that can fit in the slow-light waveguide and $N \cdot M$ is limited by the coherence time of the emitter. With our achieved device parameters, we estimate that a $3 \times 32 \mathrm{D}$ cluster state could be generated with fidelity greater than $50 \%$ (see Ref. [23] and Appendix $\mathrm{F}$ for further details). Realistic improvements in $\tau_{d}$ and $T_{2}^{*}$ could increase the size of the state by at least an order of magnitude, with even further improvement possible via incorporation of compact high kinetic inductance superconducting thin-film resonators or acoustic delay lines [57,84]. Additionally, by controlling the number of reflections a photon undergoes before exiting the metamaterial waveguide, cluster states of 3D or higher entanglement dimensionality can be generated, enabling the realization of fault-tolerant measurement-based quantum computation schemes $[23,64,85]$.

The essential paradigm of our experiment, consisting of a single artificial atom coupled to a waveguide with a long propagation delay and sharp spectral cutoffs, could, in principle, be achieved in other solid-state and atomic optical systems, such as trapped atoms coupled to a nanofiber or defect centers coupled to photonic crystal waveguides [47-50]. The challenge with such modalities, however, is achieving a large coupling of the emitter to the guided modes of the waveguide relative to its decay rate as well as the propagation delay of the waveguide. From an application standpoint, however, the optical domain is of great interest due to the mature technology in single-photon detectors, photonic integrated circuits for linear and nonlinear optics, and optical fibers for long-range communication.

\section{ACKNOWLEDGMENTS}

We thank Hannes Pichler for fruitful discussions regarding the mirror measurements, MIT Lincoln Laboratories for the provision of a traveling-wave parametric amplifier [86] used for both spectroscopic and time-domain measurements in this work, Jen-Hao Yeh and Ben Palmer for the use of one of their cryogenic attenuators [87] for reducing thermal noise in the metamaterial waveguide, and Hengjiang Ren and Xueyue Zhang for help during measurements, fabrication, and writing. This work was supported by the AFOSR MURI Quantum Photonic Matter (Grant No. 16RT0696), the Institute for Quantum Information and Matter (IQIM), an NSF Physics Frontiers Center (Grant No. PHY-1125565) with support of the Gordon and Betty Moore Foundation, and the Kavli Nanoscience Institute (KNI) at Caltech. V. S. F. gratefully acknowledges support from NSF GFRP fellowship, and M. M. (A.S.) gratefully acknowledges support from a KNI (IQIM) postdoctoral fellowship.

\section{APPENDIX A: FABRICATION AND MEASUREMENT SETUP}

\section{Device fabrication}

The devices used in this work are fabricated on $10 \mathrm{~mm} \times$ $10 \mathrm{~mm}$ silicon substrates [float zone grown, $525 \mu \mathrm{m}$ thickness, $>10 \mathrm{k} \Omega-\mathrm{cm}$ resistivity], following similar techniques as in Ref. [70]. After standard solvent cleaning of the substrate, our first aluminum (Al) layer consisting of the ground plane, CPWs, metamaterial waveguide, and qubit capacitor is patterned by electron-beam lithography of our resist followed by electron-beam evaporation of $120 \mathrm{~nm}$ aluminum at a rate of $1 \mathrm{~nm} / \mathrm{s}$. A liftoff process performed in $n$-methyl-2-pyrrolidone at $80^{\circ} \mathrm{C}$ for $2.5 \mathrm{~h}$ (with $10 \mathrm{~min}$ of ultrasonication at the end) then yields the aforementioned metal structures.

In our qubit device, the Josephson junctions are fabricated using double-angle electron beam evaporation of 
60 and $120 \mathrm{~nm}$ of $\mathrm{Al}$ (at $1 \mathrm{~nm} / \mathrm{s}$ ) on suspended Dolan bridges, with an intervening 20-min oxidation and a subsequent 2-min oxidation at $10 \mathrm{mbar}$, followed by liftoff as described above. Note that, prior to the doubleangle evaporation, the sample is cleaned by an oxygen plasma treatment and a HF vapor etch. Finally, in order to electrically connect the evaporated Josephson junctions to the first Al layer, a 6-min argon ion mill is performed to locally remove surface aluminum oxide around the areas of overlap between the first Al layer and the Josephson junctions, which is followed by evaporation of an additional "bandage" layer of $140 \mathrm{~nm} \mathrm{Al}$ that electrically connects the metal layers. Asymmetric Josephson junctions are fabricated in all qubit SQUID loops to reduce dephasing from flux noise, with a design ratio of the larger junction area to the smaller junction area of approximately 6 .

\section{Measurement setup}

A schematic of the measurement chain used in this work is shown in Fig. 5. Measurements are performed in a $3 \mathrm{He} / 4 \mathrm{He}$ dry dilution refrigerator, with a base fridge temperature at the mixing chamber (MXC) plate of $T_{f}=12 \mathrm{mK}$. The waveguide sample is wire bonded to a CPW printed circuit board (PCB) with coaxial connectors and housed inside a small copper box that is mounted to the MXC plate of the fridge. The copper box and sample are

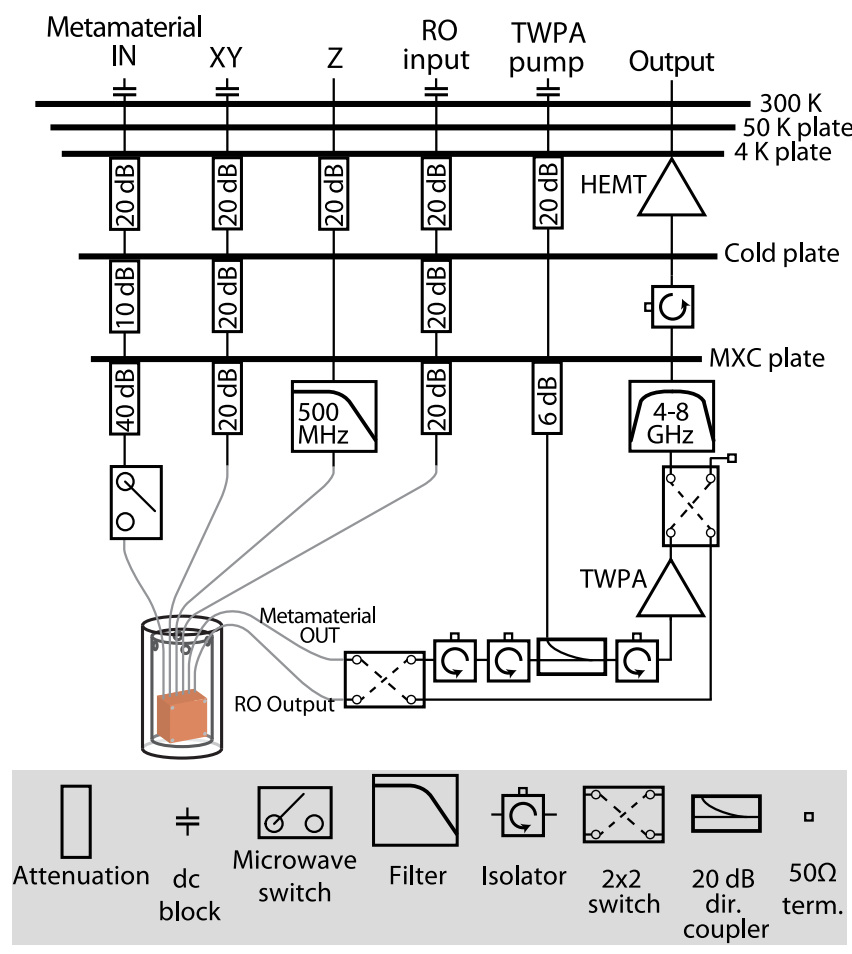

FIG. 5. Schematic of the measurement chain inside the dilution refrigerator. See Appendix text for further details ("dir" is shorthand for "directional", and "term" is shorthand for "termination"). See Fig. 2 for electrical connections at the sample. mounted inside a cryogenic magnetic shield to reduce the effects of stray magnetic field.

Attenuators are placed at several temperature stages of the fridge to provide thermalization of the coaxial input lines and to reduce thermal microwave noise at the input to the sample. We use different attenuation configurations for our gigahertz microwave lines (Metamaterial IN, $X Y$, RO input, TWPA pump) as compared to our flux line $(Z)$, with significantly less attenuation for the latter, for reasons explained in Ref. [88]. In addition, we include in the flux line a (reflective) low-pass filter, with corner frequency at $500 \mathrm{MHz}$, to minimize thermal noise photons at higher frequencies while maintaining short rise and fall time of pulses for fast flux control. Also note that the 40-dB attenuation of the "Metamaterial IN" line at the MXC plate includes a 20-dB thin-film "cold attenuator" [87] to ensure a more complete reduction of thermal photons in the metamaterial waveguide.

Our amplifier chain at the "Output" line consisted of a traveling-wave parametric amplifier (TWPA) as the initial amplification stage [86], followed by a CITCRYO4-12A high-mobility electron transistor amplifier mounted at the $4 \mathrm{~K}$ plate, and additional amplifiers at room temperature (Miteq AFS3-00101200-42-LN-HS, AMT A0262). For operation of the TWPA, a microwave pump signal is added to the amplifier via the coupled port of a $20-\mathrm{dB}$ directional coupler, with its isolated port terminated in $50 \Omega$. In between the two amplifiers, we include a reflective bandpass filter (thermalized to the MXC plate) to suppress noise outside of 4-8 GHz and use superconducting NbTi cables to minimize loss from the MXC plate to the $4 \mathrm{~K}$ plate. We also include two isolators in between the directional coupler and the sample in order to shield the sample from the strong TWPA pump, as well as an isolator in between the TWPA and the directional coupler in order to suppress any standing waves between the two elements due to spurious impedance mismatches; our isolators consist of three port circulators with the third port terminated in $50 \Omega$. All $50-\Omega$ terminations are rated for cryogenic operation and are thermalized to the MXC plate in order to suppress thermal noise from their resistive elements.

We also employ microwave switches in our measurement chain in order to provide in situ experimental flexibility in the following manner. As discussed in the main text, in between the Metamaterial IN chain and the metamaterial waveguide, we place a Radiall R573423600 microwave switch. By electrically opening the switch, we can establish an open circuit at the end of the waveguide furthest from $Q_{1}$, effectively creating a mirror for emission from $Q_{1}$ and thereby inducing time-delayed feedback.

In addition, in order to utilize our amplifier chain for either spectroscopic or time-domain measurements within the same cooldown, we employ Radiall R577432000 $2 \times 2$ microwave switches for selective routing of the outputs of the metamaterial waveguide or the readout waveguide to 
the amplification chain. With our switch configuration, we ensure that, when routing the readout waveguide output to the amplification chain, the metamaterial waveguide output is connected to a $50-\Omega$ termination. This allows us to maintain a $50-\Omega$ environment at the metamaterial output at all times and thereby ensures that the metamaterial waveguide remains an open quantum system due to its coupling to the $50-\Omega$ continuum of modes. By employing two $2 \times 2$ switches instead of one, we have the ability to bypass the TWPA amplifier if desired, although ultimately the TWPA is used when collecting all measurement data presented in Figs. 2-4.

For spectroscopic measurements, the Metamaterial IN and Output lines are connected to the input and output, respectively, of a ZNB20 Rohde \& Schwarz vector network analyzer. For time-domain measurements, gigahertz excitation and readout pulses are generated by up-conversion of megahertz IF in-phase $(I)$ and quadrature $(Q)$ signals sourced from a Keysight M320XA arbitrary waveform generator (AWG), utilizing a Marki IQ-4509 IQ mixer and a LO tone supplied by a BNC 845 microwave source. Following amplification, output readout signals are down-converted (using an equivalent mixer and the same LO source) and subsequently digitized using an Alazar ATS9360 digitizer. For all measurements, qubit flux biasing is also sourced from a M320XA AWG, the TWPA pump tone is sourced by an Agilent E8257D microwave source, and all inputs to the dilution refrigerator are low-pass filtered and attenuated such that the noise levels from the electronic sources are reduced to a $300 \mathrm{~K}$ Johnson-Nyquist noise level.

\section{APPENDIX B: CAPACITIVELY COUPLED RESONATOR ARRAY WAVEGUIDE FUNDAMENTALS}

\section{Band structure analysis}

We consider a periodic array of capacitively coupled $L C$ resonators, with the unit-cell circuit diagram shown in Fig. 1(b). The Lagrangian for this system can be constructed as a function of node fluxes $\phi_{x}$ of the resonators and is written as

$$
L=\sum_{x}\left[\frac{1}{2} C_{0} \dot{\phi}_{x}^{2}+\frac{1}{2} C_{g}\left(\dot{\phi}_{x}-\dot{\phi}_{x-1}\right)^{2}-\frac{\phi_{x}^{2}}{2 L_{0}}\right] .
$$

Since we seek traveling-wave solutions to the problem, it is convenient to work with the Fourier transform of the node fluxes, defined as

$$
\phi_{k}=\frac{1}{\sqrt{M}} \sum_{x=-N}^{N} \phi_{x} e^{-i k x d}
$$

where $M=2 N+1$ is the total number of periods of a structure with periodic boundary conditions, $d$ is the lattice constant of the resonator array, and $k$ are the discrete momenta of the first Brillouin zone's guided modes and are given by $k=(2 \pi m / M d)$ for integer $m=[-N, N]$. Using the inverse Fourier transform

$$
\phi_{x}=\frac{1}{\sqrt{M}} \sum_{k} \phi_{k} e^{i k x d}
$$

we arrive at the following $k$-space Lagrangian:

$L=\sum_{k}\left[\frac{1}{2} C_{0} \dot{\phi}_{k} \dot{\phi}_{-k}+\frac{1}{2} C_{g} \dot{\phi}_{k} \dot{\phi}_{-k}\left|1-e^{-i k d}\right|^{2}-\frac{\phi_{k} \phi_{k}}{2 L_{0}}\right]$,

where we note that $\left|1-e^{-i k d}\right|^{2}$ is equivalent to $4 \sin ^{2}(k d / 2)$. We then obtain the Hamiltonian via the standard Legendre transformation using the canonical node charges $Q_{k}=\left(\partial L / \partial \dot{\phi}_{k}\right)=\dot{\phi}_{-k}\left[C_{0}+4 C_{g} \sin ^{2}(k d / 2)\right]$, yielding

$$
H=\sum_{k}\left[\frac{1}{2} \frac{Q_{k} Q_{-k}}{\left[4 C_{g} \sin ^{2}(k d / 2)+C_{0}\right]}+\frac{\phi_{k} \phi_{-k}}{2 L_{0}}\right] .
$$

Promoting charge and flux to quantum operators and utilizing the canonical commutation relation $\left[\hat{\phi}_{k}, \hat{Q_{k^{\prime}}}\right]=$ $i \hbar \delta_{k k^{\prime}}$, we define the following creation and annihilation operators:

$$
\begin{aligned}
& \hat{a}_{k}=\sqrt{\frac{m_{k} \omega_{k}}{2 \hbar}}\left(\hat{\phi}_{k}+\frac{i}{m_{k} \omega_{k}} \hat{Q}_{-k}\right), \\
& \hat{a}_{k}^{\dagger}=\sqrt{\frac{m_{k} \omega_{k}}{2 \hbar}}\left(\hat{\phi}_{-k}+\frac{i}{m_{k} \omega_{k}} \hat{Q}_{k}\right),
\end{aligned}
$$

where $m_{k}=\left[C_{0}+4 C_{g} \sin ^{2}(k d / 2)\right]$. The resulting dispersion relation $\omega_{k}$, plotted in Fig. 1(c), is given by

$$
\omega_{k}=\frac{\omega_{0}}{\sqrt{1+4 \frac{C_{g}}{C_{0}} \sin ^{2}(k d / 2)}},
$$

where $\omega_{0}=1 / \sqrt{L_{0} C_{0}}$ and $\left[\hat{a}_{k}, \hat{a}_{k^{\prime}}^{\dagger}\right]=\delta_{k k^{\prime}}$. Expressing the flux and charge operators in terms of $\hat{a}_{k}$ and $\hat{a}_{k^{\prime}}^{\dagger}$ and substituting them into Eq. (B5), we recover the secondquantized Hamiltonian in the diagonal $k$-space basis:

$$
\hat{H}=\sum_{k} \hbar \omega_{k}\left(\frac{1}{2}+\hat{a}_{k}^{\dagger} \hat{a}_{k}\right)
$$

Note that, given the translational invariance of the capacitively coupled resonator array circuit, it is expected that the Hamiltonian would be diagonal in the Fourier plane-wave basis (Bloch theorem). 
Also note that, for two capacitively coupled $L C$ resonators, their coupling $J=\left(\omega_{0} / 2\right)\left[C_{g} /\left(C_{0}+C_{g}\right)\right]$ is positive valued [68] due to the fact that the antisymmetric odd mode of the circuit is the lower-energy eigenmode. This results in positive-valued photon hopping terms in the Hamiltonian, which directly lead to a maximum in frequency at the $\Gamma$ point and opposite directions of the phase velocity and group velocity in the structure, as observed in other dispersive media [89-91].

\section{Comparison to tight-binding model}

In the limit $C_{0} \gg C_{g}$, the dispersion is well approximated to first order by a tight-binding model with dispersion given by $\omega_{k}=\omega_{p}+2 J \cos (k d)$, where $J=$ $\omega_{0}\left(C_{g} / 2 C_{0}\right)$ is approximately the nearest-neighbor coupling between two resonators of the resonator array and $\omega_{p}=\left(\omega_{0}-2 J\right)$ is the center of the passband. The difference in the two dispersion relations reflects the coupling beyond nearest neighbor that arises due to the topology of the circuit, in which any two pairs of resonators are electrically connected through some capacitance network dependent on their distance. The magnitude of these interactions is captured in the Fourier transform of the dispersion. Consider the Fourier transform for the annihilation operator of the (localized) mode of the individual resonator located at position $x$ :

$$
\hat{a}_{k}=\frac{1}{\sqrt{M}} \sum_{x} \hat{a}_{x} e^{-i k x d} .
$$

Substituting Eq. (B9) into Eq. (B8), we arrive at the following real-space Hamiltonian:

$$
\hat{H}=\hbar \sum_{x} \sum_{x^{\prime}} V\left(x-x^{\prime}\right) \hat{a}_{x}^{\dagger} \hat{a}_{x^{\prime}},
$$

where $V\left(x-x^{\prime}\right)$ is the distance-dependent interaction strength between two resonators located at positions $x$ and $x^{\prime}$ and is simply given by the Fourier transform of the dispersion relation:

$$
V\left(x-x^{\prime}\right)=\frac{1}{M} \sum_{k} \omega_{k} e^{-i k d\left(x-x^{\prime}\right)} .
$$

For example, substituting the tight-binding dispersion $\omega_{k}=\omega_{p}+2 J \cos (k d)$ into Eq. (B11) yields $V\left(x-x^{\prime}\right)=$ $\omega_{p} \delta_{x, x^{\prime}}+2 J\left(\delta_{x-x^{\prime}, 1}+\delta_{x-x^{\prime},-1}\right)$, which, upon substitution into Eq. (B10), recovers the tight-binding Hamiltonian with only nearest-neighbor coupling.

In Fig. 6(a), we plot the magnitudes of nearest-neighbor $\left(x-x^{\prime}=1\right)$, next-nearest-neighbor $\left(x-x^{\prime}=2\right)$, and nextnext-nearest-neighbor $\left(x-x^{\prime}=3\right)$ couplings in the capacitively coupled resonator array as a function of $C_{g} / C_{0}$, calculated numerically via the discrete Fourier transform of (a)

(b)
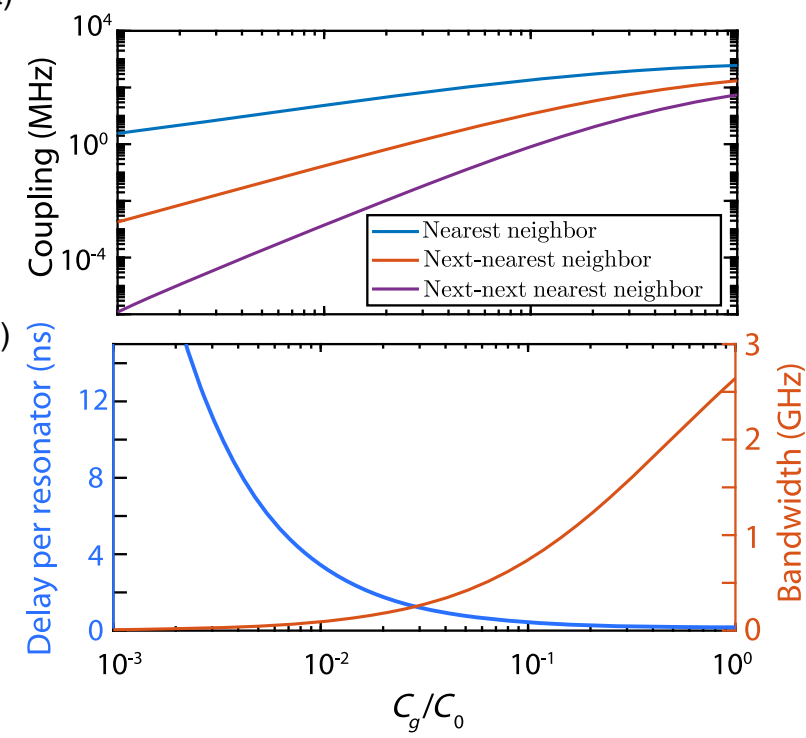

FIG. 6. (a) Magnitude of nearest-neighbor, next-nearest-neighbor, and next-next-nearest-neighbor interresonator couplings in an (infinite) capacitively coupled resonator array as a function of the $C_{g} / C_{0}$ ratio. The bare resonator frequency is chosen to be $4.8 \mathrm{GHz}$. (b) Magnitude of delay per resonator and bandwidth of the passband as a function of the $C_{g} / C_{0}$ ratio. The bare resonator frequency is again chosen to be $4.8 \mathrm{GHz}$, and the calculated delays are for frequencies in the middle of the passband.

the dispersion relation. It is evident that for small $C_{g} / C_{0}$ the nearest-neighbor coupling overwhelmingly dominates.

\section{Qubit coupled to passband of a waveguide}

The Hamiltonian of a transmonlike qubit coupled to the metamaterial waveguide via a single unit cell, where only the first two levels of the transmon $(|g\rangle,|e\rangle)$ are considered, can be written as $(\hbar=1, d=1)$

$\hat{H}=\omega_{g e}|e\rangle\langle e|+\sum_{k} \omega_{k} \hat{a}_{k}^{\dagger} \hat{a}_{k}+\frac{g_{\mathrm{uc}}}{\sqrt{M}} \sum_{k}\left(\hat{a}_{k}^{\dagger} \hat{\sigma}^{-}+\hat{a}_{k} \hat{\sigma}^{+}\right)$,

where $\omega_{k}$ is given by Eq. (B7). For an infinite array, the time-independent Schrödinger equation $\hat{H}|\psi\rangle=E|\psi\rangle$ has two types of solutions in the single-photon manifold: There are scattering eigenstates, which have an energy within the passband, and there are bound states that are energetically separated from the passband continuum. We demonstrate this in the following analysis. First, we substitute into $\hat{H}|\psi\rangle=E|\psi\rangle$ the following ansatz for the quantum states of the composite qubit-waveguide system, i.e., for dressed states of the qubit:

$$
|\psi\rangle=c_{e}|e, \mathrm{vac}\rangle+\sum_{k} c_{k} \hat{a}_{k}^{\dagger}|g, \mathrm{vac}\rangle,
$$


where $\mid$ vac $\rangle$ corresponds to no excitations in the waveguide. Doing this substitution and subsequently collecting terms, we arrive at the following coupled equations for $c_{e}$ and $c_{k}$ :

$$
\begin{gathered}
c_{e}=\frac{g_{\mathrm{uc}}}{\sqrt{M}} \sum_{k} \frac{c_{k}}{E-\omega_{g e}}, \\
c_{k}=\frac{g_{\mathrm{uc}}}{\sqrt{M}} \frac{c_{e}}{E-\omega_{k}} .
\end{gathered}
$$

By further assuming that the waveguide supports a continuum of modes (which is appropriate for a finite tapered waveguide, as described in the main text), the sum can be changed into an integral $\sum_{k} \rightarrow\left(1 / \Delta_{k}\right) \times$ $\sum_{k} \Delta_{k} \rightarrow\left(1 / \Delta_{k}\right) \int_{-\pi}^{\pi} d k$, where $\Delta_{k}=2 \pi / M$. In this continuum limit, $E$ can be found by first substituting Eq. (B15) into Eq. (B14) and subsequently dividing both sides by $c_{e}$, which yields the following transcendental equation for $E$ :

$$
E=\omega_{g e}+\frac{1}{2 \pi} \int d k \frac{g_{\mathrm{uc}}^{2}}{E-\omega_{k}},
$$

where the integral on the right-hand side of Eq. (B16) is known as the "self-energy" of the qubit $[11,13,14]$. Note that, in the opposite limit of a single resonator (where $\omega_{k}$ takes on a single value and the density of states $\partial \omega / \partial k$ becomes a delta function at that value), Eq. (B16) yields the familiar Jaynes-Cummings splitting $\sqrt{\delta^{2}+g_{\mathrm{uc}}^{2}}$.

Computation of the self-energy for $E$ such that $E>\omega_{k}$ or $E<\omega_{k} \forall k$, i.e., for energies outside of the passband, yields real solutions for Eq. (B16). On the other hand, for energies $E$ inside the passband, the self-energy integral contains a divergence at $E=\omega_{k}$ for real $E$, while there is no divergence if $E$ is allowed to be complex with an imaginary component; thus, Eq. (B16) has complex solutions when $\operatorname{Re}(E)$ is inside the passband. While a Hermitian Hamiltonian such as the one in Eq. (B12) by definition does not contain complex eigenvalues, it can be shown that the magnitude of the imaginary component of complex solutions of Eq. (B16) gives the decay rate of an excited qubit for a qubit dressed state with energy in the passband. For further details, we suggest Refs. $[8,13,14]$ to the reader. Thus, the existence of complex solutions of Eq. (B16) reflect the fact that qubit dressed states with energy in the passband are radiative states that decay into the continuum, characteristic of open quantum systems coupled to a continuum of modes. In contrast, the dressed states with (real) energies outside of the passband do not decay and are known as qubit-photon bound states in which the photonic component of the dressed-state wave function remains bound to the qubit and is not lost into the continuum.

For further analytical progress, we consider only the upper band edge and make the effective-mass approximation. This approximation is tantamount to assuming the dispersion is quadratic, such that $\omega_{k} \approx \omega_{0}-J k^{2}$, which is obtained in the limit of small $C_{g} / C_{0}$ (where $\omega_{k}$ is well approximated by the tight binding cosine dispersion) and small $k$ [where $\cos (k)$ to second order is approximately $\left.1-k^{2} / 2\right]$. This approximation is appropriate when $\omega_{g e}$ is close to the upper band edge, where the qubit is dominantly coupled to the $\Gamma$-point $k=0$ modes close to the band edge due to the van Hove singularity in the DOS, and when the lower band edge is sufficiently detuned from the qubit. Complementary analysis for the lower band edge can also be done in the same manner. For a more detailed derivation, see Refs. [7,8,13,92,93].

Under the effective-mass approximation, the self-energy integral in Eq. (B16) can be easily analyzed by taking the bounds of integration to infinity and is calculated to be $g_{\text {uc }}^{2} / 2 \sqrt{J\left(E-\omega_{0}\right)}$. For $\omega_{g e}=\omega_{0}$, Eq. (B16) then has the following two solutions:

$$
\begin{gathered}
E_{b}=\omega_{0}+\left(g_{\mathrm{uc}}^{4} / 4 J\right)^{1 / 3}, \\
E_{r}=\omega_{0}-e^{i \pi / 3}\left(g_{\mathrm{uc}}^{4} / 4 J\right)^{1 / 3} .
\end{gathered}
$$

These two solutions are indicative of a splitting of the qubit transition frequency by the band edge into two dressed states: a radiative state with energy $E_{r}$ in the passband and a bound state with energy $E_{b}$ above the band edge. The magnitude difference between the dressed-state energies is $2\left(g_{\mathrm{uc}}^{4} / 4 J\right)^{1 / 3}$, which is the frequency of coherent qubit-tophoton oscillations for an excited qubit at the photonic band edge.

For the remainder of the analysis, we focus on the qubitphoton bound state of the system. The wave function of the bound state with energy $E$ can be obtained by first substituting Eq. (15) into Eq. (B13), which yields

$$
\left|\psi_{E}\right\rangle=c_{e}\left(|e\rangle+\frac{g_{\mathrm{uc}}}{\sqrt{M}} \sum_{k} \frac{1}{E-\omega_{k}} \hat{a}_{k}^{\dagger}|g, \mathrm{vac}\rangle\right) .
$$

The qubit and photonic components of the bound state can be calculated from the normalization condition of $\left|\psi_{E}\right\rangle$ :

$$
\left|c_{e}\right|^{2}\left(1+\frac{1}{2 \pi} \int d k\left|\frac{g_{\mathrm{uc}}}{E-\omega_{k}}\right|^{2}\right)=1 .
$$

By assuming $E>\omega_{0}$, the integral in Eq. (B20) is calculated to be equal to $g_{\mathrm{uc}}^{2} / 4 \sqrt{J\left(E-\omega_{0}\right)^{3}}$, which yields the following magnitude for the qubit component of the bound state:

$$
\left|c_{e}\right|^{2}=\left(1+\frac{1}{2} \frac{E-\omega_{g e}}{E-\omega_{0}}\right)^{-1}
$$

whereas the photonic component is simply $\int d k\left|c_{k}\right|^{2}=$ $1-\left|c_{e}\right|^{2}$. We can, thus, see that, when $E \approx \omega_{g e} \neq \omega_{0}$, the qubit is negligibly hybridized with the passband modes and $\left|c_{e}\right|^{2} \approx 1$. On the other hand, as $\omega_{g e} \rightarrow \omega_{0}$ we have 
$\left|c_{e}\right|^{2} \rightarrow 2 / 3$, indicating that the bound-state photonic component contains half as much energy as the qubit component when the qubit is tuned to the band edge.

We can also obtain the real-space shape of the photonic bound state by inserting Eq. (B9) into Eq. (B19), where for a continuum of modes in $k$ space we arrive at the following photonic wave function:

$$
\sum_{x} e^{-|x| / \lambda} \hat{a}_{x}^{\dagger}|g, \mathrm{vac}\rangle
$$

up to a normalization constant, where $\lambda=\sqrt{J /\left(E-\omega_{0}\right)}$ and the qubit is assumed to reside at $x=0$. We, thus, find an exponentially localized photonic wave function for the bound state. The localization length $\lambda$ increases as $J$ increases, indicating that the bound state becomes more delocalized across multiple resonators as the strength of coupling between the resonators in the waveguide increases, whereas $\lambda$ diverges as the $E \rightarrow \omega_{0}$, which is associated with full delocalization of the bound state as its energy approaches the continuum of the passband.

\section{Group delay}

Lowering the ratio $C_{g} / C_{0}$ effectively lowers the photon hopping rate $J$ between resonators and can, thus, be chosen to significantly decrease the group velocity of propagating modes of the structure, albeit at the cost of decreased bandwidth of the passband modes. The group delay per resonator may be obtained from the inverse of the group velocity $\partial \omega_{k} / \partial k$, while the bandwidth can be calculated to be equal to $\omega_{0}\left(1-1 / \sqrt{1+4 C_{g} / C_{0}}\right)$; both are plotted in Fig. 6(b). Note that, although the group velocity approaches zero near the band edge, a traveling pulse at the band-edge frequency would experience significant distortion due to the rapidly changing magnitude of the group velocity near the band edge. At the center of the passband where the dispersion is nearly linear, however, it is possible to have propagation with minimal distortion.

Hence, in order to effectively use the coupled resonator array as a delay line, the coupling should be made sufficiently high such that the bandwidth of propagating modes (where the dispersion is also nearly linear) is sufficiently high and the effect of resonator frequency disorder due to fabrication imperfections is tolerable. After the resonator coupling constraints have been met, the desired delay may be achieved with a suitable number of resonators. It is, thus, evident that the ability to fabricate resonators of subwavelength size with minimal frequency disorder is critical to the effectiveness of implementing a slow-light waveguide with a coupled resonator array.

An appropriate metric to compare the performance of the resonator array as a delay line against dispersionless waveguides is to consider the delay achieved per area rather than per length, in order to account for the transverse dimensions of the resonators. In addition, typical implementations of delay lines with $\mathrm{CPW}$ geometries commonly require a high degree of meandering in order to fit in a packaged device; thus, the pitch and turn radius of the CPW meandered trace also must be taken into account when assessing delay achieved per area. However, by making certain simplifying assumptions about the resonators, it is possible to gain intuition on how efficient the resonator array is in achieving long delays compared to a dispersionless CPW. For the resonators implemented in the main text (see Fig. 1), the capacitive elements of the resonator are electrically connected to one end of the meander while the opposite end of the meander is shunted to ground. This geometry is, therefore, topologically similar to a $\lambda / 4$ resonator, and, consequently, the lengths of the meander and a conventional $\lambda / 4$ CPW resonator will be similar to within an order of magnitude for conventional implementations (here, $\lambda$ is the wavelength of the CPW resonator mode).

Thus, by approximating that a single resonator of the array occupies the same area as a $\lambda / 4$ section of CPW, a direct comparison between the delays of the two different waveguides can be made. In the tight-binding limit, the group delay per resonator in the middle of the passband is approximately equal to $1 / 2 J$, where $J$ is the coupling between two resonators of the array. Hence, for $N$ resonators $\tau_{d}^{\text {array }} / \tau_{d}^{\mathrm{CPW}}=[(N / 2 J) /(N \lambda / 4 v)] \sim \omega_{0} / J$, where $\tau_{d}$ is group delay and $v$ is the group velocity of light in the $\mathrm{CPW}$. Hence, the resonator array is more efficient as a delay line when compared to conventional CPW by a factor of approximately $\omega_{0} / J$ (assuming group velocity is approximately equal to phase velocity in the $\mathrm{CPW}$ ). In practice, this factor also depends on the particular geometrical implementations of both kinds of waveguide. For example, for the resonator array described in Fig. $1, \omega_{0} / J \approx 120$ and $\tau_{d}=55 \mathrm{~ns}$ delay is achieved in the middle of the passband for a resonator array of area $A=6 \mathrm{~mm}^{2}$. This constitutes a factor of 60 (500) improvement in delay per area achieved over the CPW delay line in Ref. [56] (Ref. [94]).

\section{APPENDIX C: PHYSICAL IMPLEMENTATION OF FINITE RESONATOR ARRAY}

\section{Geometrical design of unit cell}

As shown in Fig. 1, the unit cell of the resonator array in this work includes a lumped-element resonator formed from a tightly meandered wire with a large "head" capacitance and "wing" capacitors which, in addition to providing the majority of the capacitance to ground, are used to couple between resonators in neighboring unit cells. The meandered wire has a $1-\mu \mathrm{m}$ pitch and a $1-\mu \mathrm{m}$ trace width for tight packing. From the top of the meander inductor is the head capacitor and a pair of thin metal capacitor strips which extend to the lateral edges of the unit cell (the wing capacitors). The ground plane in between the resonators' meander inductor and the lateral wing capacitors acts as an electrical "fence," restricting the meander 
from coupling to neighboring resonators via stray capacitance or mutual inductance. This ensures that the bulk of the coupling between resonators is from the resonators' wing capacitive elements, thereby facilitating theoretical analysis of the structure using a simple single resonator per unit-cell model. Furthermore, we include ground metal between the thin metal capacitor traces of neighboring unitcell wing capacitors. In this way, the ground planes above and below the resonator array are tied together at each unitcell boundary, thereby suppressing the influence of higherorder transverse, slot-line modes of the waveguide.

In addition, anticipating integration with Xmon qubits, we incorporate into our unit-cell design a Xmon shunting capacitance to ground, along with pads for facile addition of Josephson junctions. This ensures that the addition of a qubit at a particular unit-cell site in the resonator array minimally affects the capacitive environment surrounding that unit cell and prevents the breaking of translational symmetry of the resonator array due to the addition of qubits. The capacitance between the Xmon capacitor and the rest of the unit cell is designed to be approximately $2 \mathrm{fF}$, yielding a qubit-unit-cell coupling of $g_{\mathrm{uc}} \approx 0.8 \mathrm{~J}$.

\section{Matching of the finite resonator array to input-output CPWs}

It has been previously shown that, for a finite coupled cavity array, low-ripple transmission at the center of the passband is possible by appropriate variation of the interresonator coupling coefficients for a few of the resonators adjacent to the ports, effectively matching the finite periodic structure to the input-output ports [95]. In the case of capacitively coupled electrical resonators, modifying the coupling capacitance in isolation results in a renormalization of the resonance frequency and, thus, constitutes a scattering center for propagating light. Thus, concurrent modification of both the coupling capacitance and the shunt capacitance to ground for the boundary resonators is necessary to achieve low-ripple transmission in the middle of the passband, as previously shown in filter design theory [96]. By constraining the total capacitance in each modified resonator to remain constant (and keeping the inductance constant), the total number of parameters to adjust in order to achieve low ripple transmission is merely equal to the chosen number of resonators to be modified, resulting in a low-dimensional optimization problem. A filter design software such as Microwave Office can be used to provide initial guesses on the optimal circuit parameters with high accuracy, which can then be further optimized.

In the main text, we present results on matching of a resonator array spanning 26 resonators to $50-\Omega$ CPWs via modification of two resonators at each of the array-CPW boundaries. The geometrical design of the boundary resonators is shown in Fig. 7. The number of boundary resonators to modify (two) is chosen as a compromise (a)

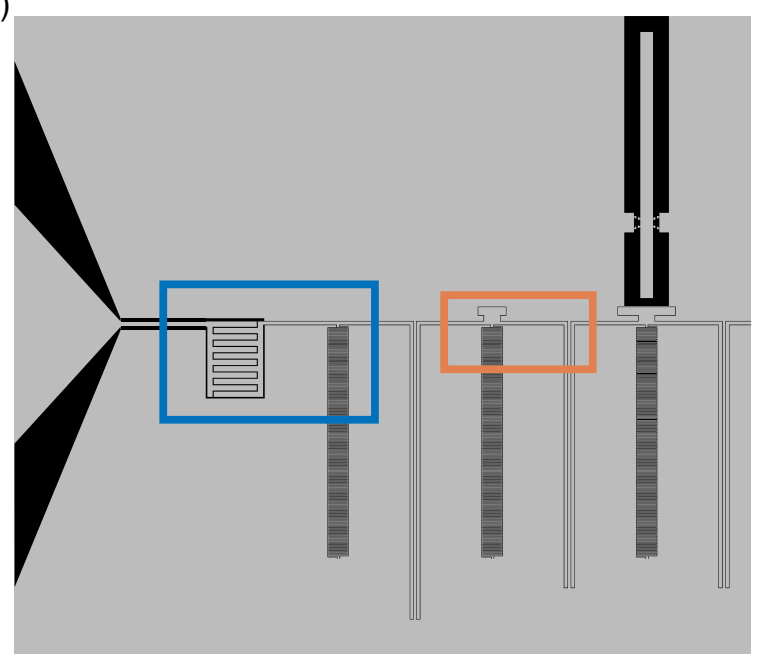

(b)

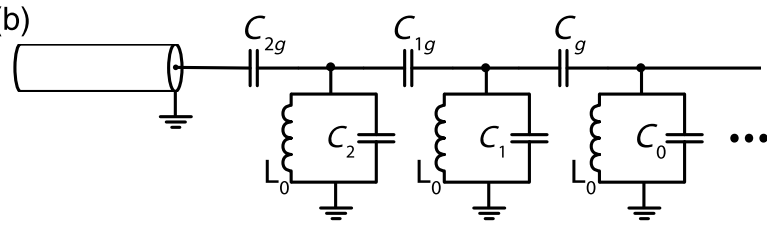

(c)
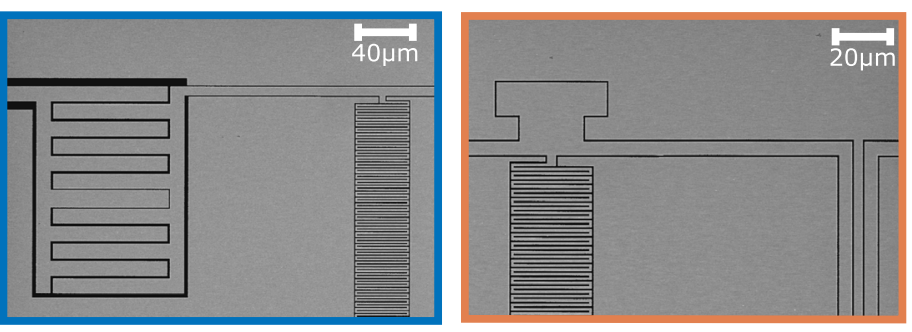

(d)

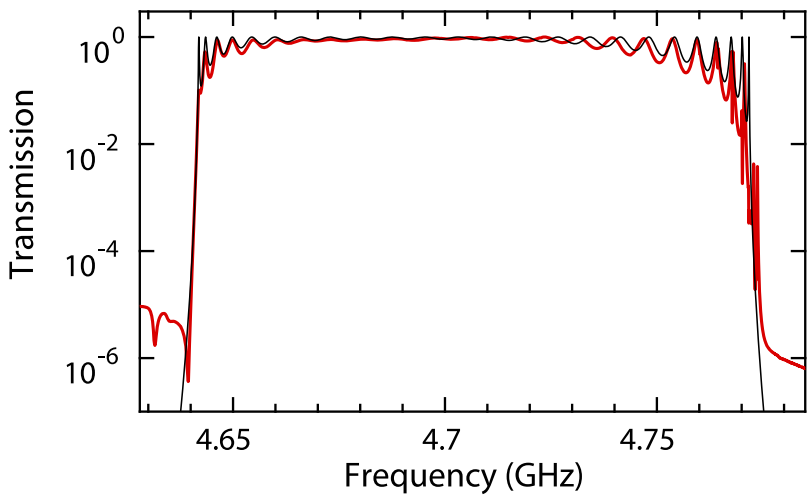

FIG. 7. (a) CAD diagram showing the end of the finite resonator array, including the boundary-matching circuit (which in this case includes the first two resonators) and the first unit cell. (b) Corresponding circuit model of the end of the finite resonator array. (c) Enlarged SEM images of the first (left) and second (right) boundary-matching resonators. (d) Transmission spectrum of the full resonator array consisting of 22 unit cells and two boundary-matching resonators on either end of the array (for a total of 26 resonators). Measured data are plotted as a red curve, and the circuit model fit is plotted as a black curve. Fit model parameters are given in the text. 
between device simplicity and spectral bandwidth over which matching occurs. In principle, however, more resonators could have been used for matching of the finite structure to the ports in order to decrease the ripples in the transmission passband near the band edges. Referring to the notation in Fig. 7(b), the targets for the unit-cell resonator and boundary resonator elements extracted from Sonnet [97] electromagnetic simulations are $C_{2 g}=89 \mathrm{fF}$, $C_{1 g}=8.9 \mathrm{fF}, C_{g}=6.47 \mathrm{fF}, C_{2}=269 \mathrm{fF}, C_{1}=351 \mathrm{fF}$, $C_{0}=353 \mathrm{fF}$, and geometric inductance $L_{0}=2.92 \mathrm{nH}$. The individual capacitive and inductive elements have parasitic inductance and capacitance, respectively, and, thus, are not simulated separately. Rather, circuit parameters for the three different resonators are extracted by simulating the whole resonator circuit. We extract the circuit element parameters from these simulations by numerically obtaining the dispersion for an infinite array of each of the three types of resonators via the $A B C D$ matrix method [69]. This yields $\omega_{0}$ and $C_{g} / C_{0} ; C_{g}$ is obtained from the $B$ parameter of the $A B C D$ matrix (which contains information on the series impedance of the unitcell circuit). We find this method of extracting parameters from simulation to give much higher accuracy when compared to other approaches, such as simulating unitcell elements separately.

Figure 7(d) shows a plot of the measured transmission spectrum of the fabricated 26-unit-cell slow-light waveguide based upon the above design and presented in the main text (cf. Fig. 1). A circuit model fit to the measured transmission spectrum yields the following circuit element parameters for boundary and central waveguide unit cells: $C_{2 g}=87.5 \mathrm{fF}, C_{1 g}=7.3 \mathrm{fF}, C_{g}=5.05 \mathrm{fF}, C_{1}=352.1 \mathrm{fF}$, $C_{2}=275.5 \mathrm{fF}, C_{0}=353.2 \mathrm{fF}$, and geometric inductance $L_{0}=3.151 \mathrm{nH}$. Based upon this model fit, we are thus able to realize good correspondence (within 3\%) between design and measured capacitances to ground, while extracted coupling capacitances are systematically lower by approximately $1.5 \mathrm{fF}$. We attribute the systematically smaller coupling to stray mutual inductance between neighboring meander inductors, which tends to lower the effective coupling impedance between the resonators. The slightly larger fit inductance compared to design is to be expected, as the kinetic inductance of the meander trace is not included in the simulation. According to Ref. [98], for a $1-\mu \mathrm{m}$ trace width and $120-\mathrm{nm}$-thick aluminum wire, the expected increase in the total inductance due to kinetic inductance is approximately $5 \%$ of the geometric inductance, in reasonable correspondence to the measured value.

\section{APPENDIX D: DISORDER ANALYSIS}

Fluctuations in the bare resonance frequencies of the lumped-element resonators making up the metamaterial waveguide break the translational symmetry of the waveguide and effectively lead to random scattering of traveling waves between different Bloch modes. This scattering results in an exponential reduction in the probability that a propagating photon traverses across the entire length of the waveguide. Furthermore, if the strength of scattering is large relative to the photon hopping rate, Anderson localization of light occurs where photons are completely trapped within the waveguide [67]. Thus, the aforementioned strategy for constructing a slow-light waveguide from an array of weakly coupled resonators is at odds with the inherit presence of fabrication disorder in any practically realizable device. Therefore, a compromise must be struck between choosing an interresonator coupling low enough to provide significant delay but high enough such that propagation through the metamaterial waveguide is not significantly compromised by resonator frequency disorder.

Figure 8(a) shows numerical calculations of the transmission extinction in the metamaterial waveguide as a function of $\sigma / J$, where $\sigma$ is the resonator frequency disorder. This analysis is performed for a 50-unit-cell waveguide, with $C_{0}=353.2 \mathrm{fF}, C_{g}=5.05 \mathrm{fF}$, and $L_{i}=$ $3.101 \mathrm{nH}+\delta_{i}$. Here, $L_{i}$ is the inductance of the $i$ th unit cell, and $\delta_{i}$ are random inductance variations in each unit cell that give rise to a particular resonator frequency disorder $\sigma$. These $L_{i}$ are calculated by (i) determining the resonator frequencies of each unit cell by drawing from a Gaussian distribution with mean $\omega_{0}$ and variance $\sigma^{2}$ and (ii) solving for the corresponding inductances given the resonator frequencies and a fixed $C_{0}$. Note that we model the disorder as originating from inductance variations, rather than $C_{0}$ or $C_{g}$ variations, based on the fact that earlier work shows that disorder in superconducting microwave resonators is primarily due to variations in kinetic inductance [99]. As we see in Fig. 8(a), in order for the average transmission to drop by less than $0.5 \mathrm{~dB}(10 \%)$, the normalized resonator frequency disorder must be less than $\sigma / J<0.1$.

In order to quantify the resonator frequency disorder in our fabricated resonator array, one can analyze the passband ripple in transmission measurements [99] [cf. Figs. 1(d) and 1(e)]. Given that the effect of tapering the circuit parameters at the boundary is to optimally couple the normal modes of the structure to the source and load impedances, the ripples in the passband are merely overlapping low- $Q$ resonances of the normal modes. Therefore, we can extract the normal mode frequencies from the maxima of the ripples in the passband, which are shifted with respect to normal mode frequencies of a structure without disorder.

Furthermore, the mode spacing is dependent on the number of resonators and, in the absence of disorder, follows the dispersion relation shown in Fig. 1(c), where the dispersion is relatively constant near the passband center and starts to shrink near the band edges. In the presence of disorder, however, this pattern breaks down as 
(a)

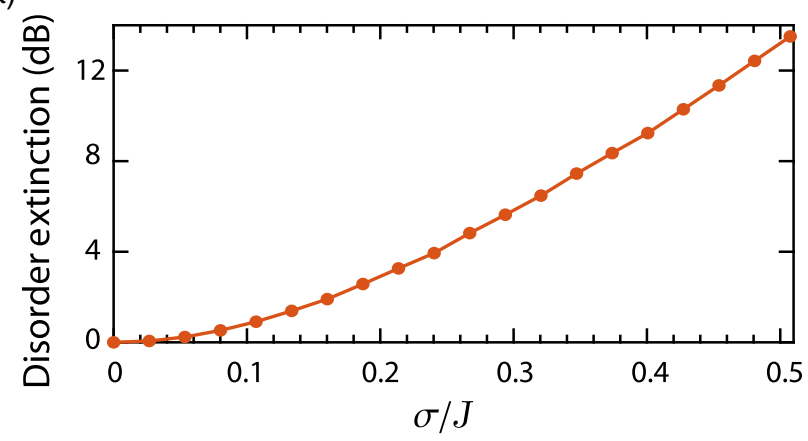

(b)

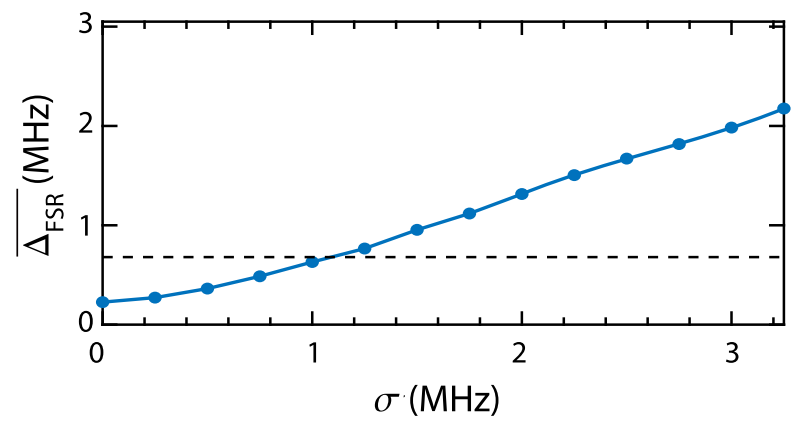

FIG. 8. (a) Numerically calculated extinction as a function of disorder. Here, $\sigma$ is the disorder in the bare frequencies of the (unit-cell) resonators making up the metamaterial waveguide, and $J$ is the coupling between nearest-neighbor resonators in the resonator array. 50 unit cells are used in this calculation, which includes taper-matching sections at the input and output of the array that bring the overall passband ripple to $0.01 \mathrm{~dB}$. For a given disorder strength $\sigma$, disorder extinction is calculated by taking the mean of the transmission across the passband for a given disorder realization and subsequently averaging that mean transmission over many disorder realizations. Note that the calculated values depend on the number of unit cells. (b) Numerically calculated variance in normal mode frequency spacing as a function of disorder. See the text for details on the method of calculation of $\overline{\Delta_{\mathrm{FSR}}}$. The dashed line indicates the experimentally measured $\Delta_{\mathrm{FSR}}$, which is extracted from the data shown in Fig. 1(d).

the modes become randomly shifted. Our approach is therefore as follows. Starting with the fit parameters presented in Appendix C, we simulate transmission through the metamaterial waveguide for varying amounts of resonator frequency disorder $\sigma$. For each level of disorder, we perform simulations of 500 different disorder realizations, and for each different disorder realization we compute the standard deviation in the free spectral range of the ripples, $\Delta_{\mathrm{FSR}}$. This deviation in free spectral range is then averaged over all disorder realizations for each value of $\sigma$, yielding an empirical relation between $\overline{\Delta_{\mathrm{FSR}}}$ and $\sigma$.

The numerically calculated empirical relation between variation in free spectral range and frequency disorder is plotted in Fig. 8(b). Note that the minimum of $\overline{\Delta_{\text {FSR }}}$ at $\sigma=0$ is set by the intrinsic dispersion of the normal mode frequencies of the unperturbed resonator array. As such, in order to yield a better sensitivity to disorder, we choose to use only the center half of the passband in our analysis where dispersion is small. From the data in Fig. 1(d), we calculate the experimental $\Delta_{\mathrm{FSR}}$. Comparing to the simulated plot in Fig. 8(b), this level of variance in the free spectral range results from a resonator frequency disorder within the array at the $1-\mathrm{MHz}$ level (or $2 \times$ $10^{-4}$ of the average resonator frequency), corresponding to $\sigma / J \approx 1 / 30$. We extract similar disorder values across a number of different metamaterial waveguide devices realized using our fabrication process.

\section{APPENDIX E: MODELING OF QUBIT $Q_{1}$ COUPLED TO THE METAMATERIAL WAVEGUIDE}

In this Appendix, we present modeling of the interaction between $Q_{1}$ and the metamaterial waveguide. Note that, while we observe dynamics that are due to emission and propagation of single-photon radiation field states, which are nonclassical states of light, in the single-excitation limit the dynamics of the qubit can also be described by a classical circuit model, where the qubit is represented by a faux resonator. Thus, here we share both viewpoints of analysis, and we employ two separate models to represent our system: a tight-binding model with nearest- and nextnearest-neighbor coupling, which we analyze via a numerical master equation solver, and a classical circuit model (shown in Fig. 10). We find excellent agreement between the two models.

\section{Tight-binding model}

\section{a. System Hamiltonian and model formalism}

For transient time-domain simulations, instead of using the Hamiltonian presented in Eq. (B12), we instead employ the following tight-binding model (with individual resonator positions denoted by the indices $x$ and $i$ ):

$$
\begin{aligned}
\hat{H}= & \omega_{g e}|e\rangle\langle e|+\sum_{x=1}^{M} \omega_{x} \hat{a}_{x}^{\dagger} \hat{a}_{x}+\left(J_{x} \hat{a}_{x}^{\dagger} \hat{a}_{x+1}\right. \\
& \left.+J_{\mathrm{nnn}} \hat{a}_{x}^{\dagger} \hat{a}_{x+2}+\text { H.c. }\right)+\sum_{i=1,3,4} g_{i} \hat{\sigma}_{x}\left(\hat{a}_{i}^{\dagger}+\hat{a}_{i}\right),
\end{aligned}
$$

where $M$ is the number of resonators, $\omega_{x}$ are the frequencies of the individual resonator modes, and, as discussed in Appendix B, in our parameter regime the capacitively coupled resonator array Hamiltonian can be well approximated as a tight-binding Hamiltonian with dominant nearest-neighbor coupling $J_{x}$ and small (approximately $J / 100$ ) next-nearest-neighbor coupling $J_{\mathrm{nnn}}$ (which we keep as a constant in the model for simplicity). In our model, for all unit cells we set $\omega_{x}=\omega_{p}=\omega_{0}-2 J$, which is the passband center frequency and constitutes the bare 
resonator frequency $\omega_{0}$ renormalized by its coupling to neighboring resonators; however, for the taper resonators, we introduce moderate detunings in order to capture the weak reflections within the slow-light waveguide evidenced by the measured data (see Fig. 3). Furthermore, we include qubit coupling to multiple resonators in the array in our model with couplings $g_{i}$, where $i$ indicates resonator position in the array, in order to capture both $g_{\mathrm{uc}}$ and the weak nonlocal coupling of the qubit to a few of the neighboring unit cells that is evidenced by the measured data.

Going into the rotating frame of the passband center frequency $\omega_{p}$ and applying the rotating wave approximation to remove counterrotating terms, we arrive at the following Hamiltonian:

$$
\begin{aligned}
\hat{H}= & \Delta_{g e}|e\rangle\langle e|+\sum_{x=1}^{M} \delta_{x} \hat{a}_{x}^{\dagger} \hat{a}_{x}+\left(J_{x} \hat{a}_{x}^{\dagger} \hat{a}_{x+1}+J_{\mathrm{nnn}} \hat{a}_{x}^{\dagger} \hat{a}_{x+2}\right. \\
& + \text { H.c. })+\sum_{i=1,3,4} g_{i}\left(\hat{a}_{i}^{\dagger} \hat{\sigma}^{-}+\hat{a}_{i} \hat{\sigma}^{+}\right),
\end{aligned}
$$

where $\Delta_{g e}=\omega_{g e}-\omega_{p}$ and $\delta_{x}=\omega_{x}-\omega_{p}$; see Fig. 9(a) for a visual diagram of the model. It can be shown that the Hamiltonian in Eq. (E2) preserves the number of (a)

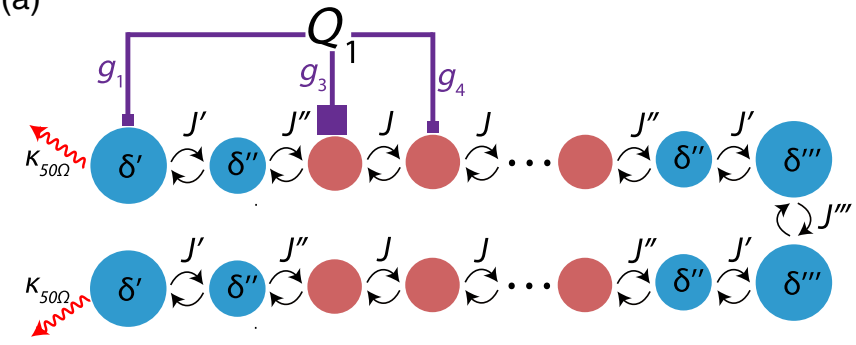

(c)

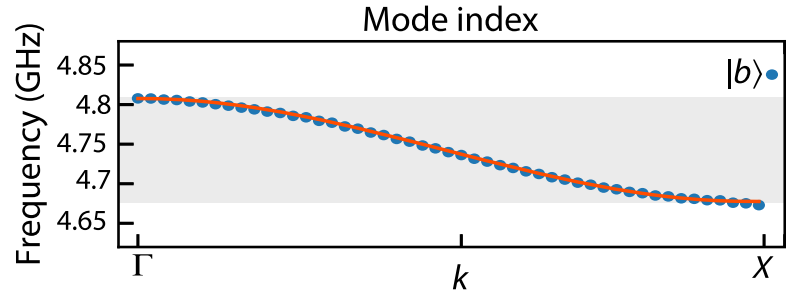

(d)
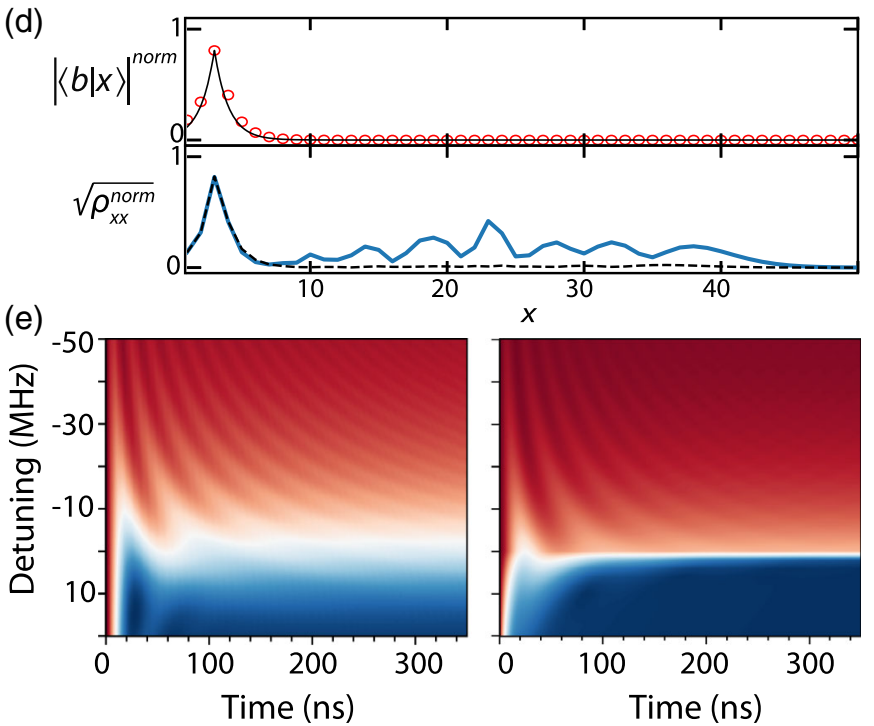

(b)

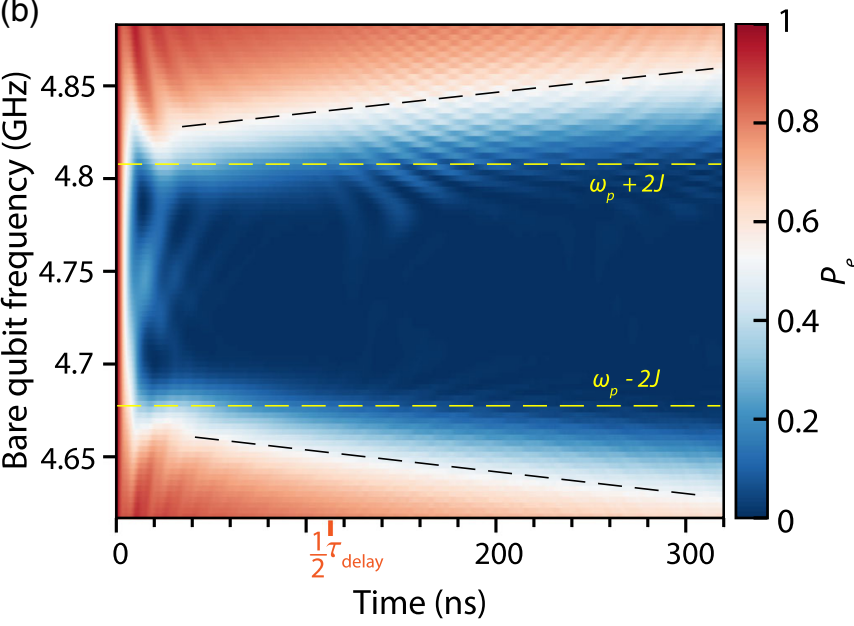

FIG. 9. Master equation numerical simulations of our qubit-slow-light waveguide system. (a) Diagram of tight-binding model used in simulations. Simulation parameters are described in the text. Note that the next-nearest-neighbor coupling $J_{\mathrm{nnn}}$, which is present in the model for all resonators, is omitted from the diagram for readability purposes. (b) Simulation of Fig. 3(b) dataset. Band edges are highlighted in dashed yellow lines, while dashed black lines are guides to the eye. (c) Scatter plot of the eigenenergies of the Hamiltonian in Eq. (E2) with $\Delta_{g e} /(2 \pi)=83 \mathrm{MHz}$ (in the single-excitation manifold) offset by $\omega_{p}$. The orange curve is a plot of the dispersion relation [see Eq. (B7)]. The eigenmode with energy outside of the passband corresponds to the bound state of the system $|b\rangle$. (d) Plot of photonic states of the system as a function of position $x$. Top: plot of the photonic wave function of the bound eigenstate of the system $|b\rangle$ in open red dots; "norm" indicates that the photonic wave function coefficients $\langle x \mid b\rangle$ are normalized by $\sqrt{\sum_{x}|\langle x \mid b\rangle|^{2}}$, where $|x\rangle$ corresponds to the state $\left|0_{1}, 0_{2}, \ldots, 1_{x}, \ldots, 0_{M} ; g\right\rangle$. The solid black line corresponds to a plot of $A e^{|x-3| / \lambda}$, where $\lambda=\sqrt{J /\left(E_{b}-\omega_{0}\right)}$ and $A$ is a normalization constant. Bottom: plot of the photonic portion of the simulated qubit-waveguide state after $t=90 \mathrm{~ns}$. The solid blue line corresponds to a simulation with initial state $\left|0_{1}, 0_{2}, \ldots, 0_{M} ; e\right\rangle$; the dashed black line corresponds to a simulation with initial state $|b\rangle . \rho_{x x}^{\text {norm }}$ refers to the scaled density matrix element $\rho_{x x} /\left(\sum_{x=1}^{10} \rho_{x x}\right)$. This particular scaling is chosen because it similarly scales the photonic part of the state within the first ten resonators of the array, thereby aiding visual comparison between the blue and dashed black curves. (e) Comparison of the dynamics simulated by a modified tight-binding model of a qubit coupled to a metamaterial waveguide (left) and by population equations of motion derived in Ref. [11] (right). Refer to (b) for the color bar. Both models assume $g_{\mathrm{uc}} / 2 \pi=19 \mathrm{MHz}$, as well as $J / 2 \pi=33 \mathrm{MHz}$. See the text for a description of the modified model. We use $\left(g_{\mathrm{uc}}^{4} / 4 J\right)^{1 / 3}$ in place of $\beta$ for simulations using Eqs. (2.21)-(2.28) from Ref. [11]. 
excitations $N$ by noting that the commutator $[\hat{H}, \hat{N}]=0$ with $\hat{N}=\sum_{x=1}^{M} \hat{a}_{x}^{\dagger} \hat{a}_{x}+\hat{\sigma}^{+} \hat{\sigma}^{-}$. Consequently, the dynamics of the system can be partitioned into subspaces with a fixed excitation number, and, for the purposes of modeling the data in Fig. 3 of a qubit's radiative dynamics in a structured photonic reservoir, we need only to consider the subspaces of $N=0,1$. The Hamiltonian in this reduced subspace can be computed by explicitly evaluating the matrix elements $\left\langle\phi|\hat{H}| \phi^{\prime}\right\rangle$ between different states $\{|\phi\rangle\}$ in the zero- and single-excitation manifold and, subsequently, directly used in numerical master equation simulations. Finally, while the Hamiltonian in Eq. (E2) generates the unitary dynamics of the system, the external loading of the system to the input-output $50-\Omega$ waveguides is incorporated into the model via dissipation with rate $\kappa_{50 \Omega}$ in the first and last resonators of the array, which is generated in our master equation simulations via collapse operators which transfer population from the single-excitation states $\left|1_{1}, 0_{2}, 0_{3}, \ldots, 0_{M} ; g\right\rangle$ and $\left|0_{1}, 0_{2}, 0_{3}, \ldots, 1_{M} ; g\right\rangle$ to the (trivial) zero-excitation ground state of the system $\left|0_{1}, 0_{2}, 0_{3}, \ldots, 0_{M}, g\right\rangle$. Note that master equation simulations of the qubit's non-Markovian radiative dynamics are possible here only due to the fact that we are explicitly simulating all the photonic degrees of freedom of the slow-light waveguide in addition to the qubit's degrees of freedom. A Lindbladian master equation simulation of solely the qubit's degrees of freedom, with the photonic degrees of freedom traced out, would not capture its non-Markovian radiative dynamics. Moreover, a simulation of the entire qubit-waveguide system is amenable here only due to our restriction of the Hilbert space to its low-energy sector and quickly grows intractable if a higher number of excitations is allowed.

Referring to Eq. (E2) and Fig. 9(a), our model assumes the following parameters $(2 \pi$ factors are omitted for readability): $M=50, \delta_{1}=\delta_{50}=\delta^{\prime}=-13.9 \mathrm{MHz}, \delta_{2}=\delta_{24}=$ $\delta_{27}=\delta_{49}=\delta^{\prime \prime}-4.7 \mathrm{MHz}, \quad \delta_{25}=\delta_{26}=\delta^{\prime \prime \prime}=323 \mathrm{MHz}$, $J_{1}=J_{24}=J_{26}=J_{49}=J^{\prime}=44.1 \mathrm{MHz}, J_{2}=J_{23}=J_{27}=$ $J_{48}=J^{\prime \prime}=32.47 \mathrm{MHz}, \quad J_{25}=J^{\prime \prime \prime}=349 \mathrm{MHz}, \quad J_{\mathrm{nnn}}=$ $0.3 \mathrm{MHz}$, all other $J_{x}=J=32.52 \mathrm{MHz}$, all other $\delta_{x}=0$, and $\kappa_{50 \Omega}=169.92 \mathrm{MHz}$ (note that the values of $\delta^{\prime \prime \prime}$ and $J^{\prime \prime \prime}$ are very different from other values in order to accurately capture the circuit of the waveguide's bend section as discussed in the main text). Note that these parameters are consistent with the circuit parameters of the model shown in Fig. 10 that is later discussed. Furthermore, in the model, we couple the qubit to the first, third, and fourth resonators of the array (as opposed to just the third resonator), with couplings $g_{1}=2.2 \mathrm{MHz}$, $g_{2}=g_{\mathrm{uc}}=26.4 \mathrm{MHz}$, and $g_{3}=3.5 \mathrm{MHz}$. Physically, the coupling to resonators 1 and 4 is not intentional and is due to parasitic capacitance. We set $g_{2}=0$ in the model, because the second metamaterial resonator is not expected to parasitically couple to the qubit as strongly as the first and fourth resonators due to the absence of an interdigitated capacitor or an integrated Xmon shunting capacitance (see Fig. 7 for images of the second resonator of the metamaterial waveguide). The $g_{1}$ and $g_{4}$ parasitic couplings are crucial to reproduce some of the subtle features in the measured data; this is discussed in detail below.

\section{b. Dynamical simulations and eigenenergy analysis}

Figure 9(b) shows the simulated dynamics from numerical master equation simulations as a function of $\Delta_{g e}$ (note that bare qubit frequency $\omega_{p}+\Delta_{g e}$ is shown in the plot instead for comparison purposes to Fig. 3) with initial state $\left|0_{1}, 0_{2}, \ldots, 0_{M} ; e\right\rangle$. It is evident that there is agreement between Fig. 9(b) and the measured data in Fig. 3(b), indicating that our model captures the salient dynamical features of our measured data. Furthermore, with the Hamiltonian in Eq. (E2), we can numerically calculate its eigenstates and the eigenenergy spectrum; as an example, the spectrum when $\Delta_{g e} /(2 \pi)=83 \mathrm{MHz}$ is plotted in Fig. 9(c). Figure 9(c) shows a band of states within the passband and a state with energy outside of the passband. Because $M=50$, the Hamiltonian is that of a finitesized system, and the band of states within the passband represent the normal modes of the finite waveguide structure; however, in the presence of input and output waveguides, they represent a band of scattering states that support wave propagation between the input and output waveguides. The state with energy outside of the band, however, is the bound eigenstate $|b\rangle$. We calculate boundstate energies as a function of bare qubit frequency $\Delta_{g e}$, and, converting bare qubit frequency to the physically applied flux through the SQUID loop used to tune the qubit frequency $\Phi$ (via measured qubit minimum and maximum frequencies and the extracted anharmonicity), we numerically obtain the predicted energy of the system's bound eigenstates as a function of flux bias and plot it in Fig. 2(d) as dashed black lines. As Fig. 2(d) shows, we obtain good quantitative agreement between the prediction of our model and the spectroscopically measured bound-state energies of the qubit-waveguide system.

In our model, the $g_{3}$ coupling primarily sets the coupling of the qubit to the metamaterial waveguide. Its magnitude relative to the $J$ between the unit cells, along with the qubit frequency $\omega_{g e}^{\prime}(\Phi)$, predominantly determines the frequency of oscillations near the band edge, as well as the decay rate into the waveguide in the passband. In the absence of other parasitic couplings, this decay rate is theoretically determined to be $\sim g_{\mathrm{uc}}^{2} / v\left[\omega_{g e}^{\prime}(\Phi)\right][92]$, where $v\left[\omega_{g e}^{\prime}(\Phi)\right]$ is the group velocity of the metamaterial waveguide at the qubitwaveguide interaction frequency $\omega_{g e}^{\prime}(\Phi)$. The parasitic coupling $g_{4}$, however, is necessary to replicate the asymmetry in the dynamics near the upper and lower band edges. This is because the lower band-edge modes have an oscillating charge distribution between unit cells, while 
the upper band-edge modes have a slowly varying charge distribution across the unit cells (which is typical of 1D tight-binding systems). The parasitic coupling of the qubit to the neighboring unit cell, therefore, has the effect of lowering the qubit coupling to the lower band-edge modes due to cancellation effects arising from the opposite charges on neighboring resonators for lower band-edge modes. On the other hand, coupling of the qubit to the upper band-edge modes which have slowly varying charge distributions is enhanced.

In addition, in simulations, the onset of oscillations seen at $\tau \approx 115 \mathrm{~ns}$ could be delayed or advanced by increasing or decreasing the number of resonators in between the qubit and the bend in the metamaterial waveguide model, while it could be removed altogether by removing the bend section. This indicates that these late-time oscillations are a result of spurious reflection of the qubit's emission at the bend, due to the imperfect matching to the 50- $\Omega$ coplanar waveguide in between the two resonator rows (which is manifested in this model through parameters $\delta^{\prime \prime \prime}$ and $\left.J^{\prime \prime \prime}\right)$. Note that this impedance mismatch and reflections are amplified near the band edges, where the Bloch impedance rapidly changes.

\section{c. Photonic state spatial analysis}

In the main text, the observed qubit emission dynamics into the slow-light waveguide are described in terms of the interplay of the qubit-waveguide dressed states, in particular, the bound and continuum dressed states of the qubit-waveguide system. Here, we further elucidate this description of our system via our modeling, using as an illustrative example the dynamics of the system when the qubit is tuned $18 \mathrm{MHz}$ above the upper band edge $\left[\Delta_{g e} /(2 \pi)=83 \mathrm{MHz}\right.$, corresponding to the brown curve in Fig. 3(c).

First, in the main text, we assert that initializing the qubit in state $|e\rangle$ with its frequency in the proximity of the passband effectively initializes it into a superposition of bound and continuum dressed states. This can be explicitly verified by first numerically calculating the eigenstates and the eigenenergy spectrum of the Hamiltonian, as is done for Fig. 9(c). As previously discussed, the state with energy outside of the band is the bound eigenstate $|b\rangle$, and the photonic component of its wave function is plotted in the top of Fig. 9(d). It is evident from Fig. 9(d) that the photonic component of the bound-state wave function is localized around resonator 3, which is the unit cell that the qubit is predominantly coupled to. As discussed in Appendix $\mathrm{B}$, the bound state is exponentially localized with localization length approximately $\lambda=\sqrt{J /\left(E_{b}-\omega_{0}\right)}$, where $E_{b}$ is the energy of the bound state; this theoretical photonic wave function is plotted in the top of Fig. 9(d) with a solid black line and shows good agreement with the numerically calculated $|b\rangle$ wave function plotted in red open dots. Numerically calculating the overlap between the $\left|0_{1}, 0_{2}, 0_{3}, \ldots, 0_{M}, e\right\rangle$ state and the bound eigenstate yields $\left|\left\langle b|| 0_{1}, 0_{2}, 0_{3}, \ldots, 0_{M}, e\right\rangle\right|^{2} \approx 0.8$, agreeing well with Eq. (B21).

Second, in the main text, we also assert that the amplitude of the early-time oscillations quickly dampens away as the energy in the radiative continuum dressed state is quickly lost into the waveguide, while the energy in the bound state remains localized around the qubit, albeit slowly decaying (details of this slow decay are given in the next paragraph). In order to illustrate this point, in the bottom of Fig. 9(d), we plot the photonic portion of the system's state at time $t=90 \mathrm{~ns}$, at which point the early-time oscillations have subsided and the qubit can be observed to be slowly decaying. It is evident that, while part of the state is delocalized in the array, a significant portion is still localized around the qubit location; this portion corresponds to the bound-state portion of the initial state $\left|0_{1}, 0_{2}, 0_{3}, \ldots, 0_{M}, e\right\rangle$ after time evolution.

Third, in order to understand the slow decay of the qubit following the early-time oscillations, note that a nonnegligible proportion of the bound-state wave function is found on resonator 1 , the taper resonator directly coupled to the output waveguide, signifying finite overlap between the bound state and the external $50-\Omega$ environment of the output waveguide. This overlap constitutes the dominant intrinsic loss channel for the bound state and leads to its slow decay, which in the $t \rightarrow \infty$ limit results in the full decay of the qubit even if its frequency is tuned outside the passband. Near the band edges, it is this loss that results in a slow population decay as compared to the initial fast dynamics in the data [see top of Fig. 3(c) for a clear example] and results in the feature highlighted by dashed black lines in Fig. 9(b). This feature would be flat for an infinite-sized resonator array, and there would be partial "population trapping" [14] of the qubit in the $t \rightarrow \infty$ limit if its bare frequency were detuned from the passband and there were no other intrinsic loss channels. Note that the $g_{1}$ coupling between the qubit and the resonator directly coupled to the $50-\Omega$ port is necessary to quantitatively replicate the slow decay rates of the qubit when its frequency is outside of the passband. In the absence of the $g_{1}$ coupling, this overlap is not sufficiently high in the simulations given the coupling of the qubit to the metamaterial waveguide (extracted from separate measurements in the passband). Therefore, this overlap is made larger, while minimizing the increase to the overall coupling of the qubit to the metamaterial waveguide, by incorporating the small $g_{1}$ coupling to the first resonator of the array.

Finally, it can be observed in Figs. 3(b) and 9 that there are differences in both duration and amplitude between the early-time oscillations and the late-time oscillations that occur at $\tau \approx 115 \mathrm{~ns}$. This is because, when the qubit frequency is near the band edges, the reflected emission is distorted through its propagation in the metamaterial waveguide due to the significant dispersion near the band edges. This results in a spatiotemporal broadening of the emitted 
radiation, which is evident in the bottom of Fig. 9(d). The frequencies of both sets of oscillations, however, are set by $g_{\mathrm{uc}}$ and $J$ as discussed in the main text.

\section{d. Comparison to paradigmatic model of spontaneous emission near the edge of a photonic band gap}

As alluded to in the main text, the early-time oscillations observed in our work are, qualitatively, a generic feature of the interaction between a qubit and a band edge in a dispersive medium and not merely an attribute of our specific system. In order to illustrate this point, in Fig. 9(e), we further compare the initial oscillations to the theory presented by John and Quang in Ref. [11] of a qubit whose frequency lies in the spectral vicinity of a band edge. The model assumed for Ref. [11] is that of an atom (qubit) with point dipole coupling to an infinite periodic dielectric environment, whose frequency is in the spectral vicinity of only a single band edge. Thus, in order to make a comparison to this theory, we change the model of our system described by Eq. (E2) and Fig. 9(a) in the following manner: (i) We remove the parasitic couplings of the qubit to neighboring unit cells, in order to simplify the coupling to a single point coupling, (ii) we increase the size of the array and move the qubit to the middle in order to remove boundary effects from the dynamics, and (iii) we reduce the overall coupling of the qubit to the metamaterial waveguide so it predominantly couples to only the band edge it is least detuned from. Note, however, that the dispersion relation of the waveguide is different than the dispersion assumed in Ref. [11]. Nonetheless, above the band edge, we see good qualitative agreement between the dynamics modeled both by the modified model and the population equation of motion derived in Ref. [11] [in particular, Eq. (2.21)], with both simulations exhibiting very similar oscillatory decay to what is observed in Figs. 9(b) and 3(b). This further confirms our interpretation of the early-time nonMarkovian dynamics in Fig. 3 discussed in the main text: that the nonexponential oscillatory decay is due to the interaction between the qubit and the strong spike in the density of states at the band edge.

\section{Circuit model}

In addition to dynamical master equation simulations, we also perform modeling via classical circuit analysis, where the qubit is represented by a linear resonator; this is an accurate representation of the qubit-waveguide system in the single-excitation limit. Time-resolved dynamical simulations are performed with the LTSpice numerical circuit simulation package, while frequency response simulations are performed with Microwave Office and standard circuit analysis. Our model, shown in Fig. 10, assumes the following metamaterial waveguide parameters: $C_{2 g}=92.5 \mathrm{fF}, C_{1 g}=7.8 \mathrm{fF}, C_{g}=5.02 \mathrm{fF}$, $C_{2}=273 \mathrm{fF}, C_{1}=351.2 \mathrm{fF}, C_{0}=353.2 \mathrm{fF}$, and $L_{0}=$ $3.099 \mathrm{nH}$, which are obtained from fitting the transmission through the metamaterial device shown in Fig. 2(a) with the qubit detuned away $(600 \mathrm{MHz})$ from the upper band edge. While, in principle, there are three independent parameters for every resonator (capacitance to ground, coupling capacitance, and inductance to ground), the set of metamaterial parameters above in addition to the qubit parameters is sufficient to achieve quantitative agreement between simulations and our data.

Our model utilizes a qubit capacitance (excluding the capacitance to the metamaterial waveguide) of $C_{\Sigma}=77.8$ $\mathrm{fF}$, which, when assuming $E_{c} \approx-\hbar \eta$, is consistent with measurements of the anharmonicity that is extracted by probing the two-photon transition between the $|g\rangle$ and $|f\rangle$ states. Furthermore, in the model we couple the qubit to the first, third, and fourth resonators of the array, with capacitive couplings $C_{1 q g}=0.16 \mathrm{fF}, C_{3 q g}=1.9 \mathrm{fF}$, and $C_{4 q g}=0.25 \mathrm{fF}$, respectively, while $C_{2 q g}=0 \mathrm{fF}$, for reproducing both the dominant and the subtle features in the measured data due to the same reasons described in the preceding discussion.

\section{a. Time domain}

Figure 10(b) shows the simulated dynamics of our circuit model as a function of the bare qubit frequency (where the qubit inductance is swept to change the bare qubit frequency). It is evident that there is agreement between Fig. 10(b) and the measured data in Fig. 3(b), indicating that our circuit model captures the salient dynamical features of our measured data. Moreover, we find excellent agreement between our circuit model and the tight-binding model presented in the preceding discussion, which is expected given that the parameters of the circuit model map nearly directly to the parameters of the tight-binding model. Thus, both models are appropriate for analyzing the data in Fig. 3, and the insights into the system gained from the tight-binding model in the preceding discussion directly carry over to this circuit model.

\section{b. Frequency domain}

In addition to time-domain simulations of our circuit model representing the fabricated qubit-waveguide system, in Fig. 10(c), we plot an intensity color plot of the transmission through the slow-light waveguide as the bare qubit frequency is tuned across the passband using the circuit model [cf. the corresponding measurement data plotted in Fig. 2(d)]. Note that, in order to capture the background transmission levels as well as the interaction of the qubit with the background transmission, we include a small direct coupling capacitance of $0.75 \mathrm{fF}$ between the first and last resonators of the array. These two resonators have the largest cross talk. This is due to the large portion of charge contained in the interdigitated capacitors between the resonators and the input-output waveguides. In simulations without this background transmission, the qubit mode breakup near the band edge and signatures of 

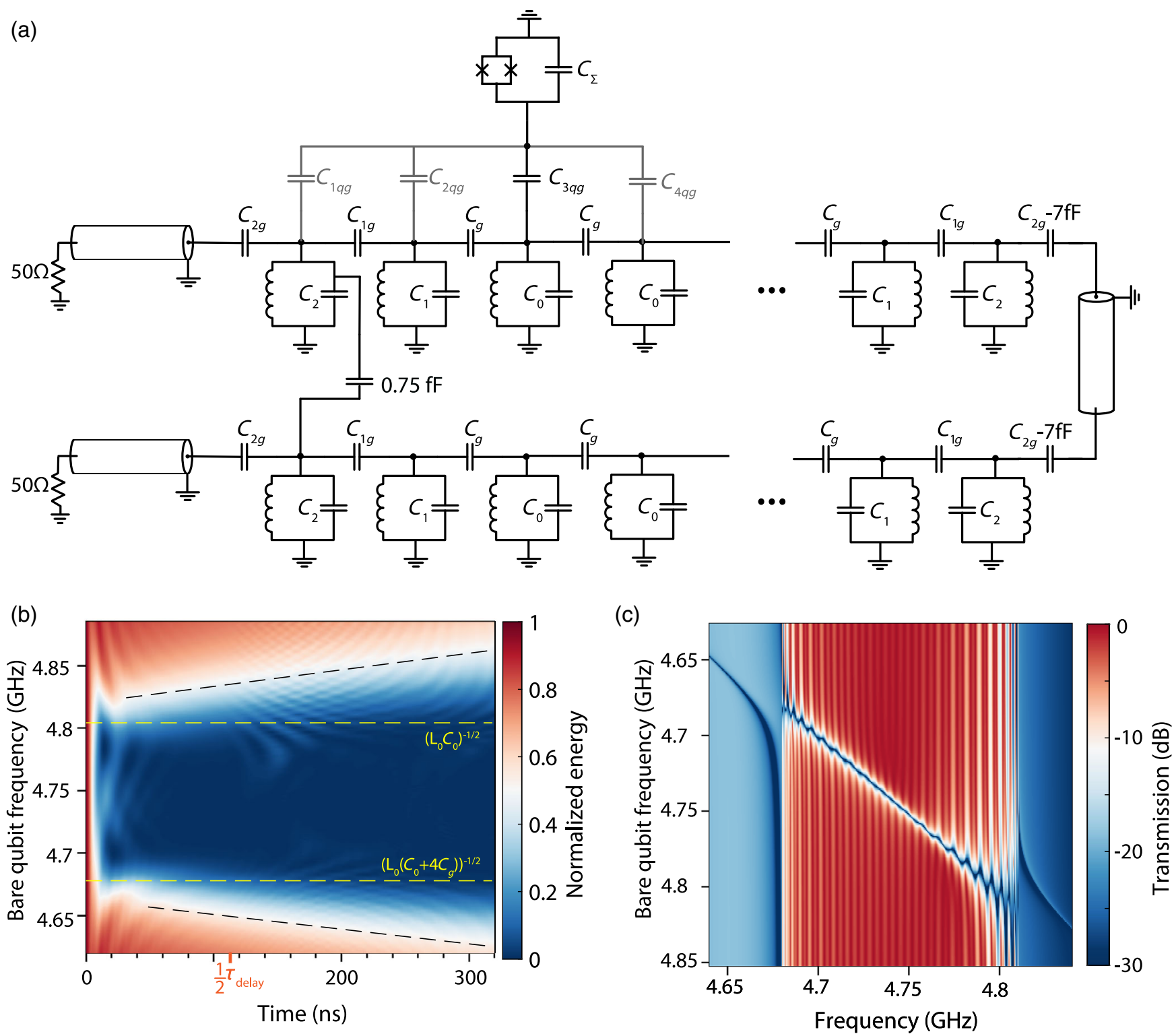

FIG. 10. (a) Full circuit model used in simulations. All inductors are made equivalent, with inductance $L_{0}$. Parameters are further discussed in the text. (b) Simulation of Fig. 3(b) dataset. The intensity plot is of energy in the faux-qubit resonator normalized by the initial energy; this simulated time-dependent normalized energy corresponds directly to the qubit's excited-state population measurements in Fig. 3(b). Simulation parameters are described in the text. Band edges are highlighted in dashed yellow lines, while dashed black lines are guides to the eye. (c) Simulation of Fig. 2(d) dataset. Circuit model and simulation parameters are described in the text. Simulations are done with the aid of the Microwave Office software package.

the bound-state outside of the passband are significantly weaker.

In addition, the series capacitance of the boundary resonators coupled to the input-output waveguides is made $7 \mathrm{fF}$ higher than the series capacitance of the boundary resonators coupled to the short CPW section in the bend, which is due to the proximity of the large bond pads used to probe the waveguides. Our simulations are in excellent qualitative agreement with the data presented in Fig. 2(d). They also capture the spectroscopic non-Markovian features of our data - the repulsion of the bound state's energy from the band edge and the persistence of the bound state even when the bare qubit frequency overlaps with the passband (see Refs. [51,52,92] for further details).

\section{APPENDIX F: UTILIZATION OF METAMATERIAL WAVEGUIDE FOR 2D CLUSTER STATE GENERATION}

We envision leveraging the large time delay and sharply varying photonic DOS of a slow-light metamaterial waveguide, along with the transmon qubit multilevel structure, 
to generate a 2D photonic cluster state. Given a typical transmon anharmonicity of $300 \mathrm{MHz}$, tuning the $e-f$ transition instead of the $g-e$ transition into the middle of the passband situates the $g-e$ transition frequency more than $200 \mathrm{MHz}$ above the upper band edge in our current waveguide devices. The corresponding level structure then consists of two metastable states $(|g\rangle$ and $|e\rangle)$ and a third level $(|f\rangle)$ that is strongly coupled to the waveguide. It has been previously shown that such a ladderlike level structure can be utilized to generate 1D cluster states of time-bin photonic qubits through a sequential emission process [62,100,101].

In addition, the non-Markovian nature of the slow-light waveguide reservoir can be further exploited to enrich this one-dimensional entanglement to higher dimensions via time-delayed feedback [23]. In the case of 2D cluster state generation, this can be accomplished by using a metamaterial waveguide terminated on one end, coupling an emitter qubit to the terminated end of the waveguide, and using a second tunable qubit coupled to the output port of the waveguide as a single-photon switchable mirror [102]. This mirror could be periodically switched on and off in a manner where consecutively emitted photons reflect on the mirror, interact a second time with the qubit, and subsequently exit through the waveguide output port without additional reflections, with facile access to the photons for subsequent measurement enabled by matching of the slow-light metamaterial waveguide to a $50-\Omega$ output waveguide. This resource-efficient scheme, requiring only two qubits, entangles photons separated in time by $\tau_{d}$ in addition to the $1 \mathrm{D}$ entanglement between consecutively emitted photons, thus achieving a $N \times M 2$ D cluster state, where $N$ is limited by the number of time-bin qubits that can fit in the slow-light waveguide and $N \cdot M$ is limited by the coherence time of the emitter. And, remarkably, increasing the number of qubit-photon interaction events by simply increasing the number of reflections in the metamaterial waveguide allows for generation of cluster states with even higher entanglement dimensionality, paving the way for fault-tolerant measurement-based quantum computation $[23,64,85]$.

Moreover, leveraging the rapid flux control of the qubit's transition frequency confers several additional advantages to the generation of multidimensional cluster states. For instance, it enables selective coupling and decoupling of the $|f\rangle$ state to the waveguide via control of the detuning of the $e-f$ transition to the passband, allowing for highfidelity manipulation of the emitter's three-level quantum state separate from photon emission and reabsorption. Additionally, controlling the qubit-waveguide interaction strength via parametric flux modulation of the qubit frequency, as discussed in the main text, allows for pulse shaping of the emitted photons [103-105], which yields multiple benefits. First, the fidelity of the photon reabsorption process can be significantly improved by shaping the photons to have a time-symmetric envelope with bandwidth less than $\Gamma_{1 \mathrm{D}}[23,75,76]$. This directly improves the fidelity of the entanglement between time-bin photonic qubits that occurs via the time-delayed feedback mechanism. Second, pulse shaping allows for precompensation of the waveguide residual dispersion near the middle of the passband [106], preventing broadening and distortion of propagating photons that could hinder their eventual measurement.

Already with our achieved device parameters of $\tau_{d}=$ $227 \mathrm{~ns}$ and $T_{2}^{*}=3 \mu \mathrm{s}$ (measured at a flux-insensitive sweet spot), along with an increased $\Gamma_{1 D}$ by a factor of 2 , entangling between individual time-bin qubits can be performed with over $95 \%$ fidelity through the techniques discussed in Ref. [23], allowing for generation of cluster states of up to approximately nine photons. Note that, due to the enhancement of the qubit-waveguide interaction strength via the slow-light effect $[107,108]$, doubling the $\Gamma_{1 \mathrm{D}}$ achieved in this work corresponds to only a small increase of approximately $2 \mathrm{fF}$ in the capacitive coupling of the qubit to the metamaterial waveguide. Furthermore, realistic increases in $\tau_{d}$ and $T_{2}^{*}$ would increase the size of possible states by at least an order of magnitude, with ample room for more substantial improvement via incorporation of even more compact high kinetic inductance superconducting thin-film resonators for larger delays, and utilization of error-protected qubits $[109,110]$ or lower-loss superconducting films [111] for higher qubit coherence. Finally, we note that techniques for tomography of microwave fields $[59,80]$, and single-photon detection of microwave photons utilizing superconducting qubits [81-83], have attained significant maturity over the past decade, enabling characterization of generated cluster states and their use in measurement-based quantum computation.

\section{APPENDIX G: MODELING OF QUBIT COUPLED TO DISPERSIONLESS WAVEGUIDE IN FRONT OF A MIRROR}

In this Appendix, we present modeling of the timedelayed feedback phenomenon described in the main text. Here, we employ a dispersionless waveguide in our model instead of our slow-light waveguide in order to compare our data to the dynamics of an ideal scenario where pulse distortion and propagation losses are absent. We employ a dispersionless waveguide with equivalent round-trip delay of $\tau_{d}=227 \mathrm{~ns}$ to the slow-light waveguide. The theoretical model we use is described at length in Ref. [31]; below, we briefly summarize the derivation of the model found in this reference.

Reference [31] starts with the following Hamiltonian, where the coupling to different waveguide modes is now allowed to vary as a function of $k$ :

$\hat{H}=\omega_{g e}|e\rangle\langle e|+\int d k \omega_{k} \hat{a}_{k}^{\dagger} \hat{a}_{k}+\int d k g_{k}\left(\hat{a}_{k}^{\dagger} \hat{\sigma}^{-}+\hat{a}_{k} \hat{\sigma}^{+}\right)$, 
and the same single-excitation ansatz of Eq. (B13), but with time-dependent coefficients $c_{e}(t)$ and $c_{k}(t)$ (and where a continuum of modes is already assumed). Following similar analysis to Appendix B, Eqs. (G1) and (B13) are substituted into the time-dependent Schrödinger equation $\partial_{t}|\psi(t)\rangle=-i \hat{H}|\psi(t)\rangle$, and, after collecting terms and going into the rotating frame of the qubit, the authors arrive at the following system of coupled differential equations:

$$
\begin{gathered}
\dot{c}_{e}(t)=-i \int d k g_{k} c_{k}(t), \\
\dot{c_{k}}(t)=-i \Delta_{k} c_{k}(t)-i g_{k} c_{e}(t),
\end{gathered}
$$

where $\Delta_{k}=\omega_{g e}-\omega_{k}$. The authors then explicitly integrate Eq. (G3) to obtain a solution for $c_{k}(t)$ and substitute that solution into Eq. (G2). In order to evaluate the resultant equation of motion for $c_{e}(t)$, the authors make the following assumptions: (i) They assume the dispersion is linearized around the qubit frequency such that $\omega_{k}=\omega_{g e}+v\left(k-k_{0}\right)$, where $v$ is the group velocity, and (ii) $g_{k}=\sqrt{\Gamma_{1 \mathrm{D}} v / \pi} \sin k x_{0}$, where $x_{0}$ is the qubit position in the waveguide. The particular form of $g_{k}$ is chosen by asserting that the field assumes a $\sin k x$ spatial profile such that the field fulfills the boundary condition of being zero at the waveguide termination; thus, the field strength at the qubit is $\sin k x_{0}$. With these expressions for $\omega_{k}$ and $g_{k}$, the resultant equation of motion for $c_{e}(t)$ can be simplified to the following form:

$\dot{c}_{e}(t)=-\frac{\Gamma_{1 \mathrm{D}}}{2} c_{e}(t)+\frac{\Gamma_{1 \mathrm{D}}}{2} e^{i 2 k_{0} x_{0}} c_{e}\left(t-\tau_{d}\right) \theta\left(t-\tau_{d}\right)$,

where $\tau_{d}$ is the round-trip delay and $\theta$ is the Heaviside step function; the first term on the right-hand side is responsible for the decay of the qubit, while the second term is responsible for photon reabsorption. Equation (G4) is finally solved via methods described in Ref. [112], yielding the following analytic expression for the dynamics of a qubit excited-state population when coupled to a semiinfinite dispersionless waveguide:

$$
\begin{aligned}
c_{e}(t)= & e^{\Gamma_{1 \mathrm{D}} t / 2} \sum_{n} \frac{1}{n !}\left(\frac{\Gamma_{1 \mathrm{D}}}{2} e^{i \phi+\Gamma_{\mathrm{ID}} \tau_{d} / 2}\right)^{n} \\
& \times\left(t-n \tau_{d}\right)^{n} \theta\left(t-n \tau_{d}\right),
\end{aligned}
$$

where $\phi=2 k_{0} x_{0}$ is the round-trip phase gained by the propagating emitted pulse.

Substituting $\Gamma_{1 \mathrm{D}} /(2 \pi)=21 \mathrm{MHz}$ and $\tau_{d}=227 \mathrm{~ns}$ into Eq. (G5), we obtain the magenta curve plotted in Fig. 4(b). As discussed in the main text, our measured dynamics compare favorably to the ideal scenario of no dispersioninduced distortion of the traveling emitted pulse, as well as

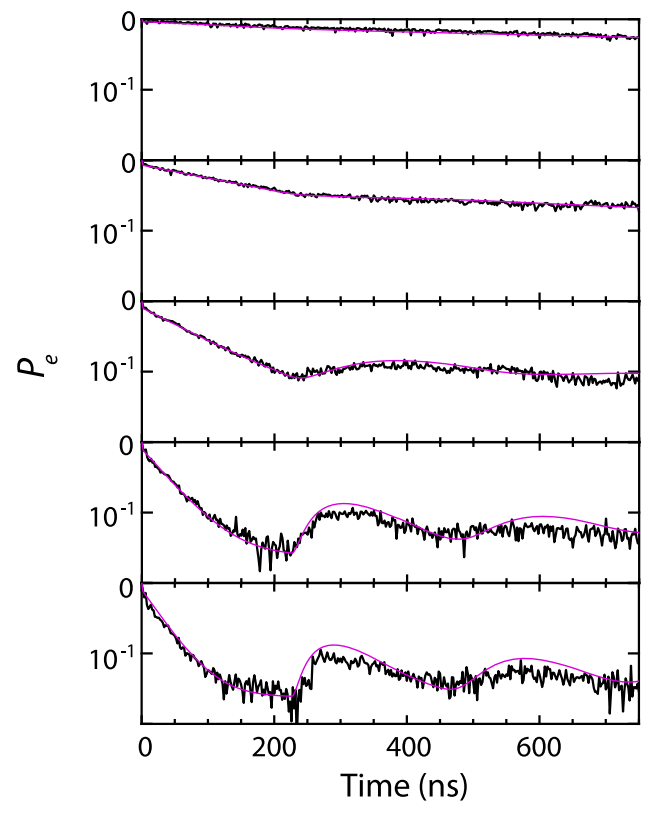

FIG. 11. Replots of the five (white) line cuts in Fig. 4(c), with accompanying theoretical predictions for emission of a qubit into a dispersionless, lossless semi-infinite waveguide. In the theoretical model, $\tau_{d}$ is maintained fixed for all simulations, while the qubit emission rate $\Gamma_{1 \mathrm{D}}$ and round-trip phase $\phi$ are allowed to vary as fit parameters to capture the effects of the changing flux-modulation amplitude, which not only changes $\Gamma_{1 \mathrm{D}}$ but also causes a residual dc shift of the average qubit frequency [77], which, in turn, affects $\phi$. Moreover, a thermal qubit population of $2.4 \%$ is assumed. From top to bottom, the fit parameters $\Gamma_{1 \mathrm{D}}$ and $\phi$ are, respectively, $\Gamma_{1 \mathrm{D}} / 2 \pi=0.17 \mathrm{MHz}, \phi=\pi / 2.6$; $\Gamma_{1 \mathrm{D}} / 2 \pi=0.6 \mathrm{MHz}, \quad \phi=\pi / 2.6 ; \quad \Gamma_{1 \mathrm{D}} / 2 \pi=1.8 \mathrm{MHz}, \quad \phi=$ $\pi / 2.1 ; \Gamma_{1 \mathrm{D}} / 2 \pi=5 \mathrm{MHz}, \phi=\pi / 2.6$. Note that the parameter $\phi$ has a negligible effect for dynamics involving large $\Gamma_{1 \mathrm{D}}$, where revival events are clearly discernible, and, for dynamics involving small $\Gamma_{1 \mathrm{D}}, \phi$ simply modulates the emission rate. However, for intermediate $\Gamma_{1 \mathrm{D}}$ such as $\Gamma_{1 \mathrm{D}} / 2 \pi=0.6$ and $1.8 \mathrm{MHz}$, the shapes of the population dynamics curves are sensitive to $\phi$.

no propagation losses, captured by the model discussed above. Thus, the limited recurrence observed can be mostly attributed to emission into the open end of the waveguide, as well as inefficient reabsorption of the emitted wave packet due to its exponential shape.

In addition, we also plot in Fig. 11 similar comparisons between this ideal model of the observed timedelayed feedback phenomenon and the data shown in Fig. 4(c). For this comparison, we choose to plot the five line cuts plotted in white in Fig. 4(c), along with comparisons to the theoretical model. The agreement between the two for all five curves is similar to the agreement observed in Fig. 4(b). Quantification of the non-Markovianity of the discussed model under various parameters is presented in Ref. [27]; however, as the reference notes, there are many competing manners to quantify non-Markovianity. 
[1] V. Weisskopf and E. Wigner, Berechnung der Natürlichen Linienbreite auf Grund der Diracschen Lichttheorie, Z. Phys. 63, 54 (1930).

[2] J. F. Haase, P. J. Vetter, T. Unden, A. Smirne, J. Rosskopf, B. Naydenov, A. Stacey, F. Jelezko, M. B. Plenio, and S. F. Huelga, Controllable Non-Markovianity for a Spin Qubit in Diamond, Phys. Rev. Lett. 121, 060401 (2018).

[3] U. Hoeppe, C. Wolff, J. Küchenmeister, J. Niegemann, M. Drescher, H. Benner, and K. Busch, Direct Observation of Non-Markovian Radiation Dynamics in 3D Bulk Photonic Crystals, Phys. Rev. Lett. 108, 043603 (2012).

[4] B.-H. Liu, L. Li, Y.-F. Huang, C.-F. Li, G.-C. Guo, E.-M. Laine, H.-P. Breuer, and J. Piilo, Experimental Control of the Transition from Markovian to NonMarkovian Dynamics of Open Quantum Systems, Nat. Phys. 7, 931 (2011).

[5] K. H. Madsen, S. Ates, T. Lund-Hansen, A. Löffler, S. Reitzenstein, A. Forchel, and P. Lodahl, Observation of Non-Markovian Dynamics of a Single Quantum Dot in a Micropillar Cavity, Phys. Rev. Lett. 106, 233601 (2011).

[6] V. P. Bykov, Spontaneous Emission from a Medium with a Band Spectrum, Sov. J. Quantum Electron. 4, 861 (1975).

[7] S. John and J. Wang, Quantum Electrodynamics near a Photonic Band Gap: Photon Bound States and Dressed Atoms, Phys. Rev. Lett. 64, 2418 (1990).

[8] S. John and J. Wang, Quantum Optics of Localized Light in a Photonic Band Gap, Phys. Rev. B 43, 12772 (1991).

[9] E. Yablonovitch, Inhibited Spontaneous Emission in SolidState Physics and Electronics, Phys. Rev. Lett. 58, 2059 (1987).

[10] S. John, Strong Localization of Photons in Certain Disordered Dielectric Superlattices, Phys. Rev. Lett. 58, 2486 (1987).

[11] S. John and T. Quang, Spontaneous Emission near the Edge of a Photonic Band Gap, Phys. Rev. A 50, 1764 (1994).

[12] H. Z. Shen, S. Xu, H. T. Cui, and X. X. Yi, Non-Markovian Dynamics of a System of Two-Level Atoms Coupled to a Structured Environment, Phys. Rev. A 99, 032101 (2019).

[13] A. González-Tudela and J. I. Cirac, Markovian and NonMarkovian Dynamics of Quantum Emitters Coupled to Two-Dimensional Structured Reservoirs, Phys. Rev. A 96, 043811 (2017).

[14] P. Lambropoulos, G. M. Nikolopoulos, T. R. Nielsen, and S. Bay, Fundamental Quantum Optics in Structured Reservoirs, Rep. Prog. Phys. 63, 455 (2000).

[15] N. Vats and S. John, Non-Markovian Quantum Fluctuations and Superradiance near a Photonic Band Edge, Phys. Rev. A 58, 4168 (1998).

[16] B. Bellomo, R. Lo Franco, and G. Compagno, NonMarkovian Effects on the Dynamics of Entanglement, Phys. Rev. Lett. 99, 160502 (2007).

[17] B. Bellomo, R. Lo Franco, S. Maniscalco, and G. Compagno, Entanglement Trapping in Structured Environments, Phys. Rev. A 78, 060302(R) (2008).

[18] C. Gonzalez-Ballestero, F. J. García-Vidal, and E. Moreno, Non-Markovian Effects in Waveguide-Mediated Entanglement, New J. Phys. 15, 073015 (2013).

[19] S. F. Huelga, A. Rivas, and M. B. Plenio, Non-MarkovianityAssisted Steady State Entanglement, Phys. Rev. Lett. 108, 160402 (2012).
[20] J. Cheng, W.-Z. Zhang, L. Zhou, and W. Zhang, Preservation Macroscopic Entanglement of Optomechanical Systems in Non-Markovian Environment, Sci. Rep. 6, 23678 (2016).

[21] D. M. Reich, N. Katz, and C. P. Koch, Exploiting NonMarkovianity for Quantum Control, Sci. Rep. 5, 12430 (2015).

[22] B. Bylicka, D. Chruściński, and S. Maniscalco, NonMarkovianity and Reservoir Memory of Quantum Channels: A Quantum Information Theory Perspective, Sci. Rep. 4, 5720 (2014).

[23] H. Pichler, S. Choi, P. Zoller, and M. D. Lukin, Universal Photonic Quantum Computation via Time-Delayed Feedback, Proc. Natl. Acad. Sci. U.S.A. 114, 11362 (2017).

[24] D. Tan, K. Ikeda, R. Saperstein, B. Slutsky, and Y. Fainman, Chip-Scale Dispersion Engineering Using Chirped Vertical Gratings, Opt. Lett. 33, 3013 (2008).

[25] C. Caloz, Metamaterial Dispersion Engineering Concepts and Applications, Proc. IEEE 99, 1711 (2011).

[26] A. Säynätjoki, M. Mulot, J. Ahopelto, and H. Lipsanen, Dispersion Engineering of Photonic Crystal Waveguides with Ring-Shaped Holes, Opt. Express 15, 8323 (2007).

[27] T. Tufarelli, M.S. Kim, and F. Ciccarello, NonMarkovianity of a Quantum Emitter in Front of a Mirror, Phys. Rev. A 90, 012113 (2014).

[28] H. Pichler and P. Zoller, Photonic Circuits with Time Delays and Quantum Feedback, Phys. Rev. Lett. 116, 093601 (2016).

[29] I.-C. Hoi, A. Kockum, L. Tornberg, A. Pourkabirian, G. Johansson, P. Delsing, and C. Wilson, Probing the Quantum Vacuum with an Artificial Atom in Front of a Mirror, Nat. Phys. 11, 1045 (2015).

[30] Y.-L. L. Fang, F. Ciccarello, and H. U. Baranger, NonMarkovian Dynamics of a Qubit due to Single-Photon Scattering in a Waveguide, New J. Phys. 20, 043035 (2018).

[31] T. Tufarelli, F. Ciccarello, and M. S. Kim, Dynamics of Spontaneous Emission in a Single-End Photonic Waveguide, Phys. Rev. A 87, 013820 (2013).

[32] D. O. Krimer, M. Liertzer, S. Rotter, and H. E. Türeci, Route from Spontaneous Decay to Complex Multimode Dynamics in Cavity QED, Phys. Rev. A 89, 033820 (2014).

[33] A. Carmele, J. Kabuss, F. Schulze, S. Reitzenstein, and A. Knorr, Single Photon Delayed Feedback: A Way to Stabilize Intrinsic Quantum Cavity Electrodynamics, Phys. Rev. Lett. 110, 013601 (2013).

[34] L. Guo, A. F. Kockum, F. Marquardt, and G. Johansson, Oscillating Bound States for a Giant Atom, Phys. Rev. Research 2, 043014 (2020).

[35] F. Dinc, Diagrammatic Approach for Analytical NonMarkovian Time Evolution: Fermi's Two-Atom Problem and Causality in Waveguide Quantum Electrodynamics, Phys. Rev. A 102, 013727 (2020).

[36] G. Calajó, Y.-L. L. Fang, H. U. Baranger, and F. Ciccarello, Exciting a Bound State in the Continuum through Multiphoton Scattering plus Delayed Quantum Feedback, Phys. Rev. Lett. 122, 073601 (2019).

[37] S. Garmon, K. Noba, G. Ordonez, and D. Segal, NonMarkovian Dynamics Revealed at a Bound State in the Continuum, Phys. Rev. A 99, 010102(R) (2019). 
[38] F. Dinc and A. M. Brańczyk, Non-Markovian SuperSuperradiance in a Linear Chain of up to 100 Qubits, Phys. Rev. Research 1, 032042(R) (2019).

[39] K. Sinha, P. Meystre, E. A. Goldschmidt, F. K. Fatemi, S. L. Rolston, and P. Solano, Non-Markovian Collective Emission from Macroscopically Separated Emitters, Phys. Rev. Lett. 124, 043603 (2020).

[40] H. Zheng and H. U. Baranger, Persistent Quantum Beats and Long-Distance Entanglement from Waveguide-Mediated Interactions, Phys. Rev. Lett. 110, 113601 (2013).

[41] A. Carmele, N. Nemet, V. Canela, and S. Parkins, Pronounced Non-Markovian Features in Multiply Excited, Multiple Emitter Waveguide QED: Retardation Induced Anomalous Population Trapping, Phys. Rev. Research 2, 013238 (2020).

[42] T. Ramos, B. Vermersch, P. Hauke, H. Pichler, and P. Zoller, Non-Markovian Dynamics in Chiral Quantum Networks with Spins and Photons, Phys. Rev. A 93, 062104 (2016).

[43] R. J. Schoelkopf and S. M. Girvin, Wiring up Quantum Systems, Nature (London) 451, 664 (2008).

[44] M. H. Devoret and R. J. Schoelkopf, Superconducting Circuits for Quantum Information: An Outlook, Science 339, 1169 (2013).

[45] A. F. Van Loo, A. Fedorov, K. Lalumière, B. C. Sanders, A. Blais, and A. Wallraff, Photon-Mediated Interactions between Distant Artificial Atoms, Science 342, 1494 (2013).

[46] K. Lalumiere, B. C. Sanders, A. F. van Loo, A. Fedorov, A. Wallraff, and A. Blais, Input-Output Theory for Waveguide QED with an Ensemble of Inhomogeneous Atoms, Phys. Rev. A 88, 043806 (2013).

[47] E. Vetsch, D. Reitz, G. Sagué, R. Schmidt, S. T. Dawkins, and A. Rauschenbeutel, Optical Interface Created by Laser-Cooled Atoms Trapped in the Evanescent Field Surrounding an Optical Nanofiber, Phys. Rev. Lett. 104, 203603 (2010).

[48] S.-P. Yu, J. Hood, J. Muniz, M. Martin, R. Norte, C.-L. Hung, S. M. Meenehan, J. D. Cohen, O. Painter, and H. Kimble, Nanowire Photonic Crystal Waveguides for Single-Atom Trapping and Strong Light-Matter Interactions, Appl. Phys. Lett. 104, 111103 (2014).

[49] A. Javadi, I. Söllner, M. Arcari, S. L. Hansen, L. Midolo, S. Mahmoodian, G. Kiršanskè, T. Pregnolato, E. Lee, J. Song et al., Single-Photon Non-linear Optics with a Quantum Dot in a Waveguide, Nat. Commun. 6, 8655 (2015).

[50] M. K. Bhaskar, D. D. Sukachev, A. Sipahigil, R. E. Evans, M. J. Burek, C. T. Nguyen, L. J. Rogers, P. Siyushev, M. H. Metsch, H. Park et al., Quantum Nonlinear Optics with a Germanium-Vacancy Color Center in a Nanoscale Diamond Waveguide, Phys. Rev. Lett. 118, 223603 (2017).

[51] N. M. Sundaresan, R. Lundgren, G. Zhu, A. V. Gorshkov, and A. A. Houck, Interacting Qubit-Photon Bound States with Superconducting Circuits, Phys. Rev. X 9, 011021 (2019).

[52] Y. Liu and A. A. Houck, Quantum Electrodynamics near a Photonic Bandgap, Nat. Phys. 13, 48 (2017).

[53] G. Andersson, B. Suri, L. Guo, T. Aref, and P. Delsing, Non-exponential Decay of a Giant Artificial Atom, Nat. Phys. 15, 1123 (2019).
[54] M. Mirhosseini, E. Kim, V. S. Ferreira, M. Kalaee, A. Sipahigil, A. J. Keller, and O. Painter, Superconducting Metamaterials for Waveguide Quantum Electrodynamics, Nat. Commun. 9,3706 (2018).

[55] M. Mirhosseini, E. Kim, X. Zhang, A. Sipahigil, P. B. Dieterle, A. J. Keller, A. Asenjo-Garcia, D. E. Chang, and O. Painter, Cavity Quantum Electrodynamics with Atomlike Mirrors, Nature (London) 569, 692 (2019).

[56] Y. P. Zhong, H.-S. Chang, K. J. Satzinger, M.-H. Chou, A. Bienfait, C. R. Conner, É. Dumur, J. Grebel, G. A. Peairs, R. G. Povey et al., Violating Bell's Inequality with Remotely Connected Superconducting Qubits, Nat. Phys. 15, 741 (2019).

[57] A. Bienfait, K. J. Satzinger, Y. Zhong, H.-S. Chang, M.-H. Chou, C. Conner, É. Dumur, J. Grebel, G. Peairs, R. Povey et al., Phonon-Mediated Quantum State Transfer and Remote Qubit Entanglement, Science 364, 368 (2019).

[58] I.-C. Hoi, T. Palomaki, J. Lindkvist, G. Johansson, P. Delsing, and C. M. Wilson, Generation of Nonclassical Microwave States Using an Artificial Atom in 1D Open Space, Phys. Rev. Lett. 108, 263601 (2012).

[59] C. Eichler, C. Lang, J. M. Fink, J. Govenius, S. Filipp, and A. Wallraff, Observation of Entanglement between Itinerant Microwave Photons and a Superconducting Qubit, Phys. Rev. Lett. 109, 240501 (2012).

[60] J. Koch, T. M. Yu, J. Gambetta, A. A. Houck, D. I. Schuster, J. Majer, A. Blais, M. H. Devoret, S. M. Girvin, and R. J. Schoelkopf, Charge-Insensitive Qubit Design Derived from the Cooper Pair Box, Phys. Rev. A 76, 042319 (2007).

[61] R. Barends, J. Kelly, A. Megrant, D. Sank, E. Jeffrey, Y. Chen, Y. Yin, B. Chiaro, J. Mutus, C. Neill, P. O’Malley, P. Roushan, J. Wenner, T. C. White, A. N. Cleland, and J. M. Martinis, Coherent Josephson Qubit Suitable for Scalable Quantum Integrated Circuits, Phys. Rev. Lett. 111, 080502 (2013).

[62] C. Schön, E. Solano, F. Verstraete, J. I. Cirac, and M. M. Wolf, Sequential Generation of Entangled Multiqubit States, Phys. Rev. Lett. 95, 110503 (2005).

[63] A. Russo, E. Barnes, and S. E. Economou, Generation of Arbitrary All-Photonic Graph States from Quantum Emitters, New J. Phys. 21, 055002 (2019).

[64] S. Xu and S. Fan, Generate Tensor Network State by Sequential Single-Photon Scattering in Waveguide QED Systems, APL Photonics 3, 116102 (2018).

[65] A. Yariv, Y. Xu, R. K. Lee, and A. Scherer, CoupledResonator Optical Waveguide: A Proposal and Analysis, Opt. Lett. 24, 711 (1999).

[66] M. Notomi, E. Kuramochi, and T. Tanabe, LargeScale Arrays of Ultrahigh-q Coupled Nanocavities, Nat. Photonics 2, 741 (2008).

[67] D. S. Wiersma, P. Bartolini, A. Lagendijk, and R. Righini, Localization of Light in a Disordered Medium, Nature (London) 390, 671 (1997).

[68] S. M. Girvin, in Quantum Machines: Measurement and Control of Engineered Quantum Systems, edited by M. Devoret, B. Huard, R. Schoelkopf, and L. F. Cugliandolo (Oxford University Press, New York, 2011), Chap. 3, pp. 113-256.

[69] D. M. Pozar, Microwave Engineering (Wiley, New York, 2009). 
[70] A. J. Keller, P. B. Dieterle, M. Fang, B. Berger, J. M. Fink, and O. Painter, Al Transmon Qubits on Silicon-onInsulator for Quantum Device Integration, Appl. Phys. Lett. 111, 042603 (2017).

[71] S. Shevchenko, S. Ashhab, and F. Nori, Landau-ZenerStückelberg Interferometry, Phys. Rep. 492, 1 (2010).

[72] E.-M. Laine, J. Piilo, and H.-P. Breuer, Measure for the Non-Markovianity of Quantum Processes, Phys. Rev. A 81, 062115 (2010).

[73] H.-P. Breuer, Foundations and Measures of Quantum NonMarkovianity, J. Phys. B 45, 154001 (2012).

[74] G. S. Agarwal, Vacuum-Field Rabi Oscillations of Atoms in a Cavity, J. Opt. Soc. Am. B 2, 480 (1985).

[75] Y. Wang, J. Minár, L. Sheridan, and V. Scarani, Efficient Excitation of a Two-Level Atom by a Single Photon in a Propagating Mode, Phys. Rev. A 83, 063842 (2011).

[76] M. Stobińska, G. Alber, and G. Leuchs, Perfect Excitation of a Matter Qubit by a Single Photon in Free Space, Europhys. Lett. 86, 14007 (2009).

[77] J. Li, M. Silveri, K. Kumar, J.-M. Pirkkalainen, A. Vepsäläinen, W. Chien, J. Tuorila, M. Sillanpää, P. Hakonen, E. Thuneberg et al., Motional Averaging in a Superconducting Qubit, Nat. Commun. 4, 1420 (2013).

[78] S. Barrett, K. Hammerer, S. Harrison, T. E. Northup, and T.J. Osborne, Simulating Quantum Fields with Cavity QED, Phys. Rev. Lett. 110, 090501 (2013).

[79] C. Eichler, J. Mlynek, J. Butscher, P. Kurpiers, K. Hammerer, T. J. Osborne, and A. Wallraff, Exploring Interacting Quantum Many-Body Systems by Experimentally Creating Continuous Matrix Product States in Superconducting Circuits, Phys. Rev. X 5, 041044 (2015).

[80] C. Eichler, D. Bozyigit, C. Lang, L. Steffen, J. Fink, and A. Wallraff, Experimental State Tomography of Itinerant Single Microwave Photons, Phys. Rev. Lett. 106, 220503 (2011).

[81] S. Kono, K. Koshino, Y. Tabuchi, A. Noguchi, and Y. Nakamura, Quantum Non-demolition Detection of an Itinerant Microwave Photon, Nat. Phys. 14, 546 (2018).

[82] J.-C. Besse, S. Gasparinetti, M. C. Collodo, T. Walter, P. Kurpiers, M. Pechal, C. Eichler, and A. Wallraff, SingleShot Quantum Nondemolition Detection of Individual Itinerant Microwave Photons, Phys. Rev. X 8, 021003 (2018).

[83] A. L. Grimsmo, B. Royer, J. M. Kreikebaum, Y. Ye, K. O'Brien, I. Siddiqi, and A. Blais, Quantum Metamaterial for Broadband Detection of Single Microwave Photons, Phys. Rev. Applied 15, 034074 (2021).

[84] A. Shearrow, G. Koolstra, S. J. Whiteley, N. Earnest, P. S. Barry, F. J. Heremans, D. D. Awschalom, E. Shirokoff, and D. I. Schuster, Atomic Layer Deposition of Titanium Nitride for Quantum Circuits, Appl. Phys. Lett. 113, 212601 (2018).

[85] R. Raussendorf, J. Harrington, and K. Goyal, Topological Fault-Tolerance in Cluster State Quantum Computation, New J. Phys. 9, 199 (2007).

[86] C. Macklin, K. O’Brien, D. Hover, M. E. Schwartz, V. Bolkhovsky, X. Zhang, W. D. Oliver, and I. Siddiqi, A Near-Quantum-Limited Josephson Traveling-Wave Parametric Amplifier, Science 350, 307 (2015).
[87] J.-H. Yeh, J. LeFebvre, S. Premaratne, F. Wellstood, and B. Palmer, Microwave Attenuators for Use with Quantum Devices below 100 mk, J. Appl. Phys. 121, 224501 (2017).

[88] S. Krinner, S. Storz, P. Kurpiers, P. Magnard, J. Heinsoo, R. Keller, J. Luetolf, C. Eichler, and A. Wallraff, Engineering Cryogenic Setups for 100-Qubit Scale Superconducting Circuit Systems, Eur. Phys. J. Quantum Technol. 6, 2 (2019).

[89] H. Gersen, T. J. Karle, R. J. P. Engelen, W. Bogaerts, J. P. Korterik, N. F. van Hulst, T. F. Krauss, and L. Kuipers, Direct Observation of Bloch Harmonics and Negative Phase Velocity in Photonic Crystal Waveguides, Phys. Rev. Lett. 94, 123901 (2005).

[90] Y. Wang, Y. Zhang, L. He, F. Liu, H. Li, and H. Chen, Direct Observation of Negative Phase Velocity and Positive Group Velocity in Time Domain for Composite Right/Left-Handed Transmission Lines, J. Appl. Phys. 100, 113503 (2006).

[91] J. Woodley and M. Mojahedi, Backward Wave Propagation in Left-Handed Media with Isotropic and Anisotropic Permittivity Tensors, J. Opt. Soc. Am. B 23, 2377 (2006).

[92] G. Calajó, F. Ciccarello, D. Chang, and P. Rabl, AtomField Dressed States in Slow-Light Waveguide QED, Phys. Rev. A 93, 033833 (2016).

[93] F. Lombardo, F. Ciccarello, and G. M. Palma, Photon Localization versus Population Trapping in a CoupledCavity Array, Phys. Rev. A 89, 053826 (2014).

[94] Y. Wang, H. T. Su, F. Huang, and M. J. Lancaster, WideBand Superconducting Coplanar Delay Lines, IEEE Trans. Microwave Theory Tech. 53, 2348 (2005).

[95] M. Sumetsky and B. J. Eggleton, Modeling and Optimization of Complex Photonic Resonant Cavity Circuits, Opt. Express 11, 381 (2003).

[96] S. B. Cohn, Direct-Coupled-Resonator Filters, Proc. IRE 45, 187 (1957).

[97] Sonnet, Sonnet Suites, Version 16.52.

[98] J. Gao, The Physics of Superconducting Microwave Resonators, Ph.D. thesis, California Institute of Technology, 2008.

[99] D. L. Underwood, W. E. Shanks, J. Koch, and A. A. Houck, Low-Disorder Microwave Cavity Lattices for Quantum Simulation with Photons, Phys. Rev. A 86, 023837 (2012).

[100] I. Schwartz, D. Cogan, E. R. Schmidgall, Y. Don, L. Gantz, O. Kenneth, N. H. Lindner, and D. Gershoni, Deterministic Generation of a Cluster State of Entangled Photons, Science 354, 434 (2016).

[101] J.-C. Besse, K. Reuer, M. C. Collodo, A. Wulff, L. Wernli, A. Copetudo, D. Malz, P. Magnard, A. Akin, M. Gabureac, G. J. Norris, J. I. Cirac, A. Wallraff, and C. Eichler, Realizing a Deterministic Source of MultipartiteEntangled Photonic Qubits, Nat. Commun. 11, 4877 (2020).

[102] J.-T. Shen and S. Fan, Coherent Single Photon Transport in a One-Dimensional Waveguide Coupled with Superconducting Quantum Bits, Phys. Rev. Lett. 95, 213001 (2005).

[103] A. N. Korotkov, Flying Microwave Qubits with Nearly Perfect Transfer Efficiency, Phys. Rev. B 84, 014510 (2011).

[104] Y. Yin, Y. Chen, D. Sank, P. J. J. O’Malley, T. C. White, R. Barends, J. Kelly, E. Lucero, M. Mariantoni, A. Megrant 
et al., Catch and Release of Microwave Photon States, Phys. Rev. Lett. 110, 107001 (2013).

[105] P. Forn-Diaz, C. W. Warren, C. W. S. Chang, A. M. Vadiraj, and C.M. Wilson, On-Demand Microwave Generator of Shaped Single Photons, Phys. Rev. Applied 8, 054015 (2017).

[106] R. I. Killey, P. M. Watts, M. Glick, and P. Bayvel, in Proceedings of the 2006 Optical Fiber Communication Conference and the National Fiber Optic Engineers Conference (IEEE, New York, 2006).

[107] S. Hughes, Enhanced Single-Photon Emission from Quantum Dots in Photonic Crystal Waveguides and Nanocavities, Opt. Lett. 29, 2659 (2004).

[108] P. Lodahl, S. Mahmoodian, and S. Stobbe, Interfacing Single Photons and Single Quantum Dots with Photonic Nanostructures, Rev. Mod. Phys. 87, 347 (2015).
[109] A. Kitaev, Protected Qubit Based on a Superconducting Current Mirror, arXiv:cond-mat/0609441.

[110] P. W. Shor, Scheme for Reducing Decoherence in Quantum Computer Memory, Phys. Rev. A 52, R2493 (1995).

[111] A. P. Place, L. V. Rodgers, P. Mundada, B. M. Smitham, M. Fitzpatrick, Z. Leng, A. Premkumar, J. Bryon, A. Vrajitoarea, S. Sussman, G. Cheng, T. Madhavan, H. K. Babla1, X. H. Le, Y. Gang, B. Jäck, A. Gyenis, N. Yao, R. J. Cava, N. P. de Leon, and A. A. Houck, New Material Platform for Superconducting Transmon Qubits with Coherence Times Exceeding 0.3 milliseconds, Nat. Commun. 12, 1779 (2021).

[112] H. T. Dung and K. Ujihara, Analytic Solution for Retardation in Two-Atom Systems, Phys. Rev. A 59, 2524 (1999). 